S UPPORTING INFORMATION FOR:

\title{
Nanosecond Relaxation Dynamics of Protein GB1 Identified by the Time-Dependent Red Shift in the Fluorescence of Tryptophan and 5-Fluorotryptophan
}

\author{
Dmitri Toptygin, ${ }^{\S}$ Angela M. Gronenborn, ${ }^{\mathbb{T}}$ and Ludwig Brand ${ }^{\S}$ \\ §Department of Biology, Johns Hopkins University, Baltimore, MD, USA. \\ "Laboratory of Chemical Physics, NIDDK, National Institutes of Health,Bethesda, MD, \\ USA. Current address: Department of Structural Biology, University of Pittsburgh School \\ of Medicine, Pittsburgh, PA, USA.
}

\section{Contents}

Figure 1S. Schematic diagram of the experimental setup for spectrally-resolved time-correlated photon

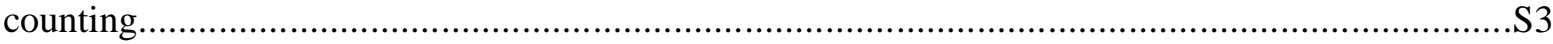

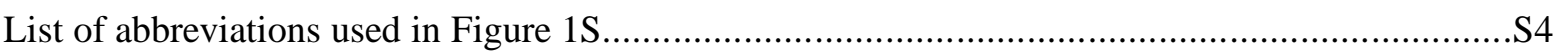

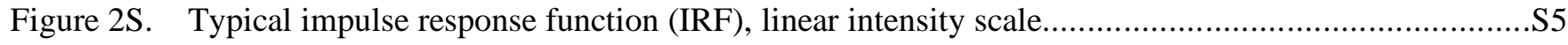

Figure 3S. Typical impulse response function (IRF), logarithmic intensity scale......................................S6

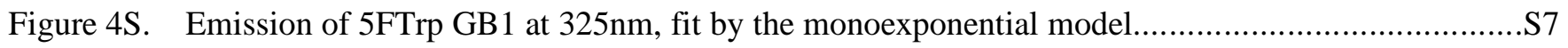

Figure 5S. Emission of 5FTrp GB1 at 325nm, fit by the double-exponential model..............................S8

Figure 6S. Emission of 5FTrp GB1 at 325nm, fit by the triple-exponential model.....................................S9

Figure 7S. Emission of 5FTrp GB1 at 325nm, fit by the quadruple-exponential model...........................S10

Figure 8S. Emission of 5FTrp GB1 at 325nm, fit by the quintuple-exponential model...............................S11

Figure 9S. Emission of 5FTrp GB1 at 355nm, fit by the monoexponential model..................................... 12

Figure 10S. Emission of 5FTrp GB1 at 355nm, fit by the double-exponential model...............................S13

Figure 11S. Emission of 5FTrp GB1 at 355nm, fit by the triple-exponential model.................................S 14

Figure 12S. Emission of 5FTrp GB1 at 355nm, fit by the quadruple-exponential model...........................S15

Figure 13S. Emission of 5FTrp GB1 at 355nm, fit by the quintuple-exponential model................................S16

Figure 14S. Emission of 5FTrp GB1 at 390nm, fit by the monoexponential model..................................S17

Figure 15S. Emission of 5FTrp GB1 at 390nm, fit by the double-exponential model..................................S18

Figure 16S. Emission of 5FTrp GB1 at 390nm, fit by the triple-exponential model..................................S19

Figure 17S. Emission of 5FTrp GB1 at 390nm, fit by the quadruple-exponential model............................S20

Figure 18S. Emission of 5FTrp GB1 at 390nm, fit by the quintuple-exponential model.............................S21

Figure 19S. Emission of unmodified GB1 at 320nm, fit by the monoexponential model..........................S22

Figure 20S. Emission of unmodified GB1 at 320nm, fit by the double-exponential model.........................S23

Figure 21S. Emission of unmodified GB1 at 320nm, fit by the triple-exponential model...........................S24

Figure 22S. Emission of unmodified GB1 at 320nm, fit by the quadruple-exponential model......................S25

Figure 23S. Emission of unmodified GB1 at 320nm, fit by the quintuple-exponential model......................S26

Figure 24S. Emission of unmodified GB1 at 350nm, fit by the monoexponential model...........................S27

Figure 25S. Emission of unmodified GB1 at 350nm, fit by the double-exponential model.........................S28

Figure 26S. Emission of unmodified GB1 at 350nm, fit by the triple-exponential model..........................S29

Figure 27S. Emission of unmodified GB1 at $350 \mathrm{~nm}$, fit by the quadruple-exponential model......................S30

Figure 28S. Emission of unmodified GB1 at 350nm, fit by the quintuple-exponential model.....................S31

Figure 29S. Emission of unmodified GB1 at $385 \mathrm{~nm}$, fit by the monoexponential model............................S32

Figure 30S. Emission of unmodified GB1 at 385nm, fit by the double-exponential model..........................S33 
Figure 31S. Emission of unmodified GB1 at $385 \mathrm{~nm}$, fit by the triple-exponential model...........................S34

Figure 32S. Emission of unmodified GB1 at 385nm, fit by the quadruple-exponential model......................S35

Figure 33S. Emission of unmodified GB1 at $385 \mathrm{~nm}$, fit by the quintuple-exponential model......................S36

Table 1S. Best-fit parameters for 5FTrp GB1 emission at 325nm, quadruple-exponential model...............S37

Table 2S. Best-fit parameters for 5FTrp GB1 emission at 355nm, double-exponential model..................S37

Table 3S. Best-fit parameters for 5FTrp GB1 emission at 390nm, triple-exponential model.....................S37

Table 4S. Best-fit parameters for unmodified GB1 emission at 320nm, quadruple-exponential model.......S38

Table 5S. Best-fit parameters for unmodified GB1 emission at 350nm, double-exponential model............S38

Table 6S. Best-fit parameters for unmodified GB1 emission at $385 \mathrm{~nm}$, triple-exponential model.............S38

Figure 34S. Fluorescence kinetics of 5FTrp and Trp in GB1, presented in the format of Fig.1 in ref. 24.........S39

Figure 35S. The dynamics of the time-dependent red shift for Trp and 5FTrp, without the $t \rightarrow \infty$ limit

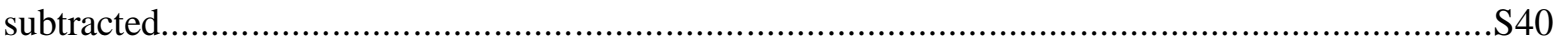

Parametrization of the functions $S(v)$ and $N(t)$ involved in the model function in Eq. (8)..............................S41

Figure 36S. Color map of global weighted residuals for the stretched exponential relaxation model..............S43

Figure 37S. Color map of global weighted residuals for the triple-exponential relaxation model...................S43 
Figure 1S. Schematic diagram of the experimental setup for spectrally-resolved timecorrelated photon counting.

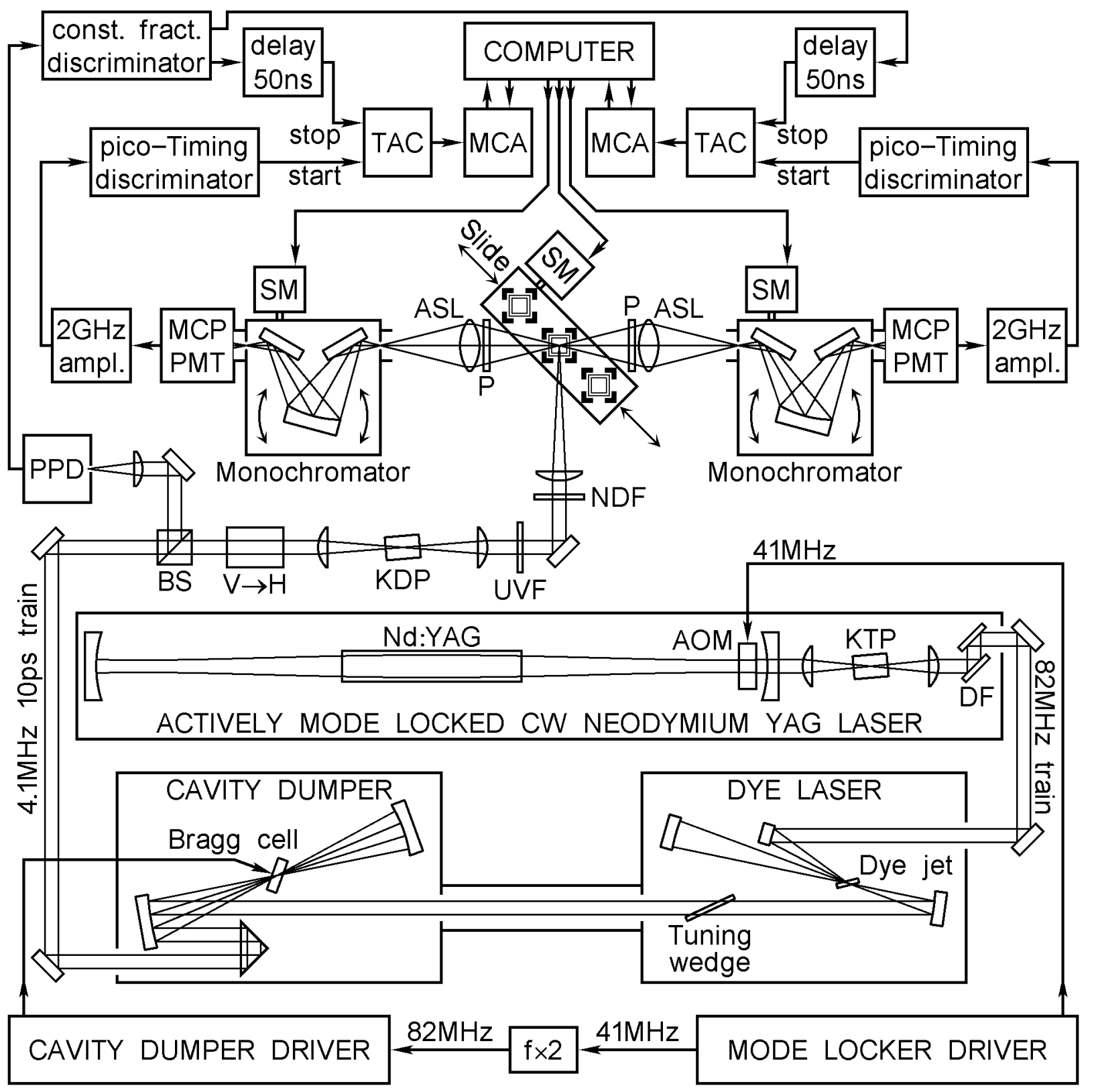




\section{List of abbreviations used in Figure 1S.}

2GHz ampl. Low-noise linear amplifier, $1.8 \mathrm{GHz}$ bandpass, gain $=50$, input \& output impedance $50 \Omega$. Attached directly to the output of MCP-PMT.

ACTIVELY MODE LOCKED CW NEODYMIUM YAG LASER Spectra-Physics model 3460.

AOM Acousto-Optical Modulator.

ASL ASpheric condenser Lenses, quartz (Oriel, 50.0mm diameter).

BS Beam Splitter; diverts 1\% to PPD, transmits $99 \%$ straight through.

CAVITY DUMPER Spectra-Physics model 344.

CAVITY DUMPER DRIVER Spectra-Physics model 454.

const. fract. discriminator Constant fraction discriminator (Tennelec model TC 454).

DF Dichroic Filter, consists of two dichroic mirrors with high reflectivity at 532nm and low reflectivity at $1064 \mathrm{~nm}$ (for $45^{\circ}$ angle of incidence).

DYE LASER Spectra-Physics model 375B.

$\mathrm{f} \times 2 \quad$ Electronic frequency doubler. Converts $41 \mathrm{MHz}$ synchronization output of the mode locker driver to $82 \mathrm{MHz}$. The $82 \mathrm{MHz}$ synchronization signal is fed to the photodiode input of the cavity dumper driver. The frequency doubler replaces the photodiode in the cavity dumper and considerably improves the stability of the laser system.

KDP Potassium dihydrophosphate nonlinear optical crystal (Inrad model 562-126). Frequency-doubles the dye laser output $(572 \ldots 638 \mathrm{~nm})$ to UV radiation $(286 \ldots 319 \mathrm{~nm})$.

KTP Potassium thallium phosphate nonlinear optical crystal for frequency-doubling the infrared Nd:YAG laser output (1064nm) to visible radiation (532nm).

MCA MultiChannel Analyzer (EG\&G Ortec ADCAM 918).

MCP-PMT MicroChannel Plate PhotoMulTiplier (Hamamatsu R1564U-06). Mounted directly onto the output slit of the monochromator.

MODE LOCKER DRIVER Spectra-Physics models 453 and 452A.

Monochromator Jobin Yvon H10-UV diffraction grating monochromator, motor-driven.

NDF Neutral Density Filter, adjustable. Decreases exciting UV power from $\sim 500 \mu \mathrm{W}$ to $<5 \mu \mathrm{W}$. Helps to avoid both protein photobleaching and photon pile-up.

Nd:YAG Neodymium-doped Yttrium-Aluminum Garnet crystal, the optical gain medium.

$\mathrm{P} \quad$ Polarizers in the emission path (Oriel 20LP-UV, 50.8mm diameter). For total intensity measurements the polarizers are set at $55^{\circ}$ from the vertical (the magic angle).

pico-Timing discriminator EG\&G Ortec pico-Timing ${ }^{\mathrm{TM}}$ discriminator 9307.

PPD Picosecond PhotoDetector, a passivated non-avalanche silicon PIN photodiode with multi-GHz bandpass (Antel model AR-S2).

Slide A linear translational stage with three thermostatted cuvette holders.

SM Synchronous Motors (Slo-Syn $\left.{ }^{\circledR}\right)$, computer-controlled via the computer serial port. Three SloSyn ${ }^{\circledR}$ motors drive two Monochromators and one Slide.

TAC Time-to-Amplitude Converter (EG\&G Ortec model 457), custom modified to suppress the crosstalk between "start" and "stop" inputs.

$\mathrm{V} \rightarrow \mathrm{H} \quad$ A broadband polarization rotator (Oriel), converting Vertical polarization to Horizontal polarization. The frequency doubler shown to the right of this polarization rotator converts horizontally-polarized visible radiation to vertically-polarized UV radiation.

UVF UltraViolet Filter (Corning 7-54). Blocks visible light, transmits UV radiation. 
Figure 2S. Typical impulse response function (IRF), linear intensity scale.

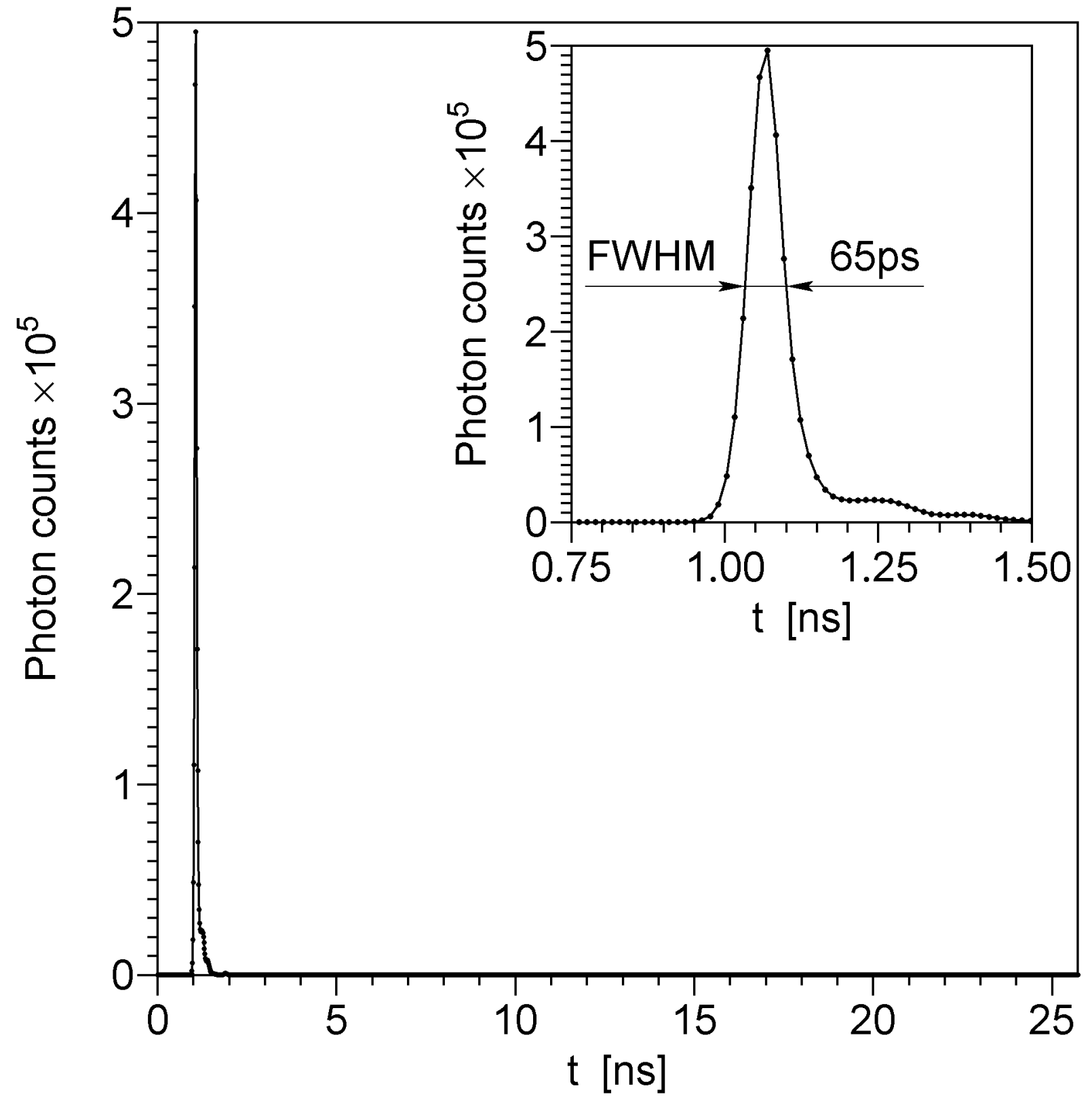

An IRF is measured using scattering Ludox® (colloid silica) suspension in water. This IRF was measured quasi-simultaneously with the fluorescence emission of unmodified Trp GB1 at 320nm and was actually used in the analysis of the $320 \mathrm{~nm}$ data. The data analysis program calculated a numerical convolution of the IRF with the multiexponential model function from Eq.(2) and fitted the convolution to the fluorescence emission data. The inset shows the same IRF on 16x expanded time scale, which makes it possible to see the actual width of the IRF at half maximum. 
Figure 3S. Typical impulse response function (IRF), logarithmic intensity scale.

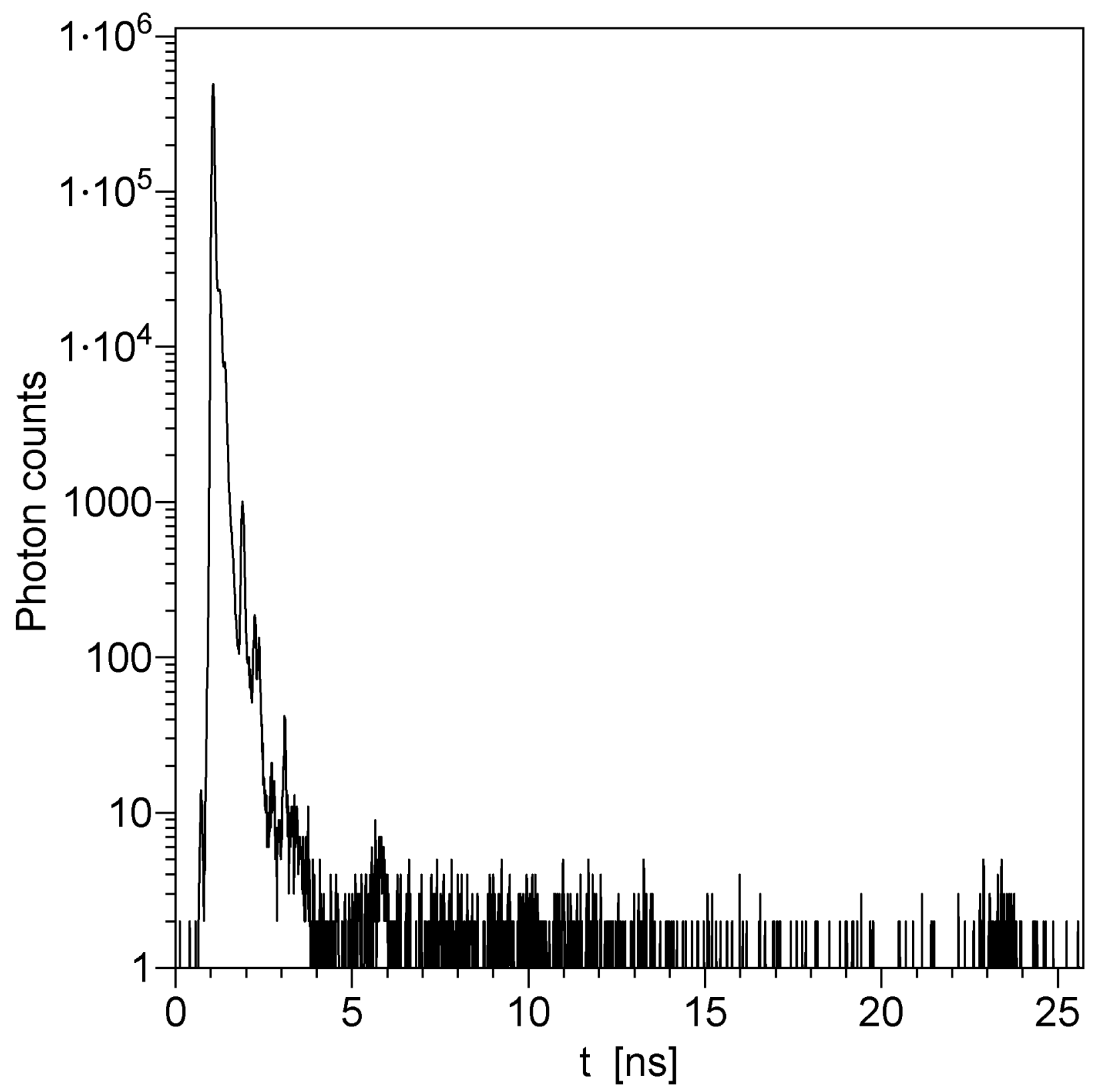

This representation better reveals small-intensity satellite peaks, which are due to reflections of light from miscellaneous optical elements. In our experimental setup the highest satellite peak has about $1 / 500$ of the intensity of the main IRF peak, thus, on the linear intensity scale the satellite peaks are invisible. 
Figure 4S. Emission of 5FTrp GB1 at $325 \mathrm{~nm}$, fit by the monoexponential model.

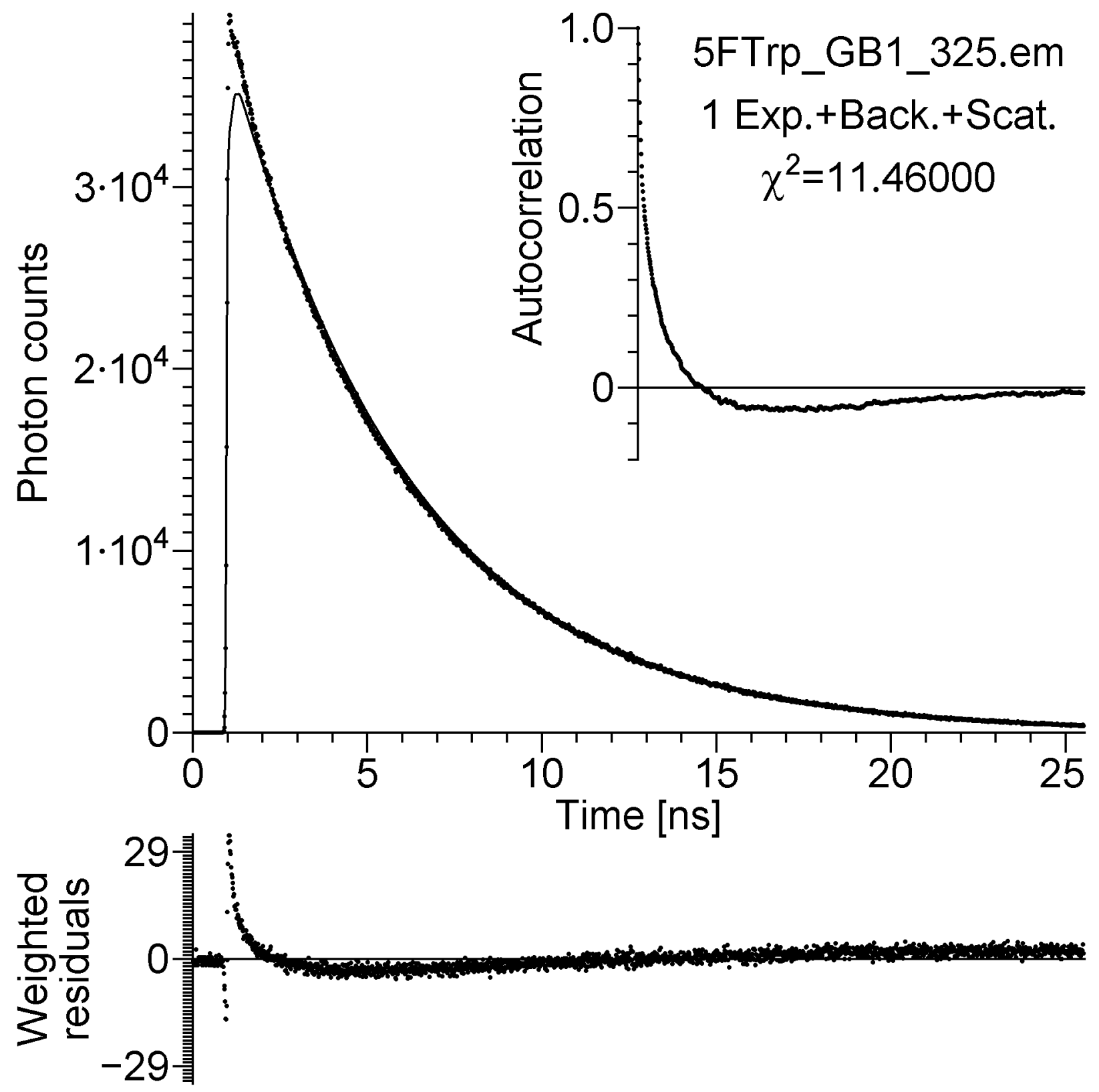


Figure 5S. Emission of 5FTrp GB1 at 325nm, fit by the double-exponential model.

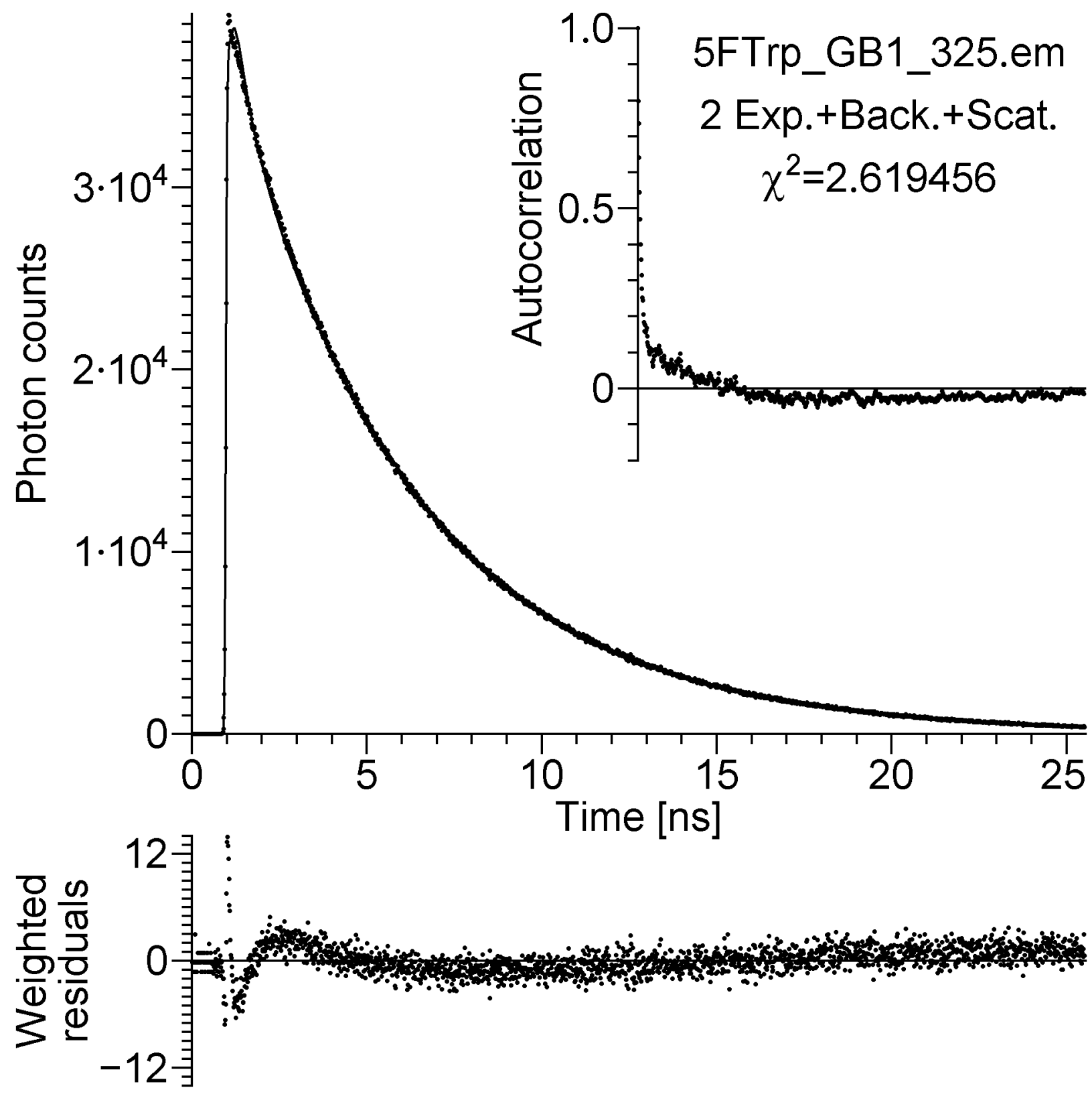


Figure 6S. Emission of 5FTrp GB1 at 325nm, fit by the triple-exponential model.
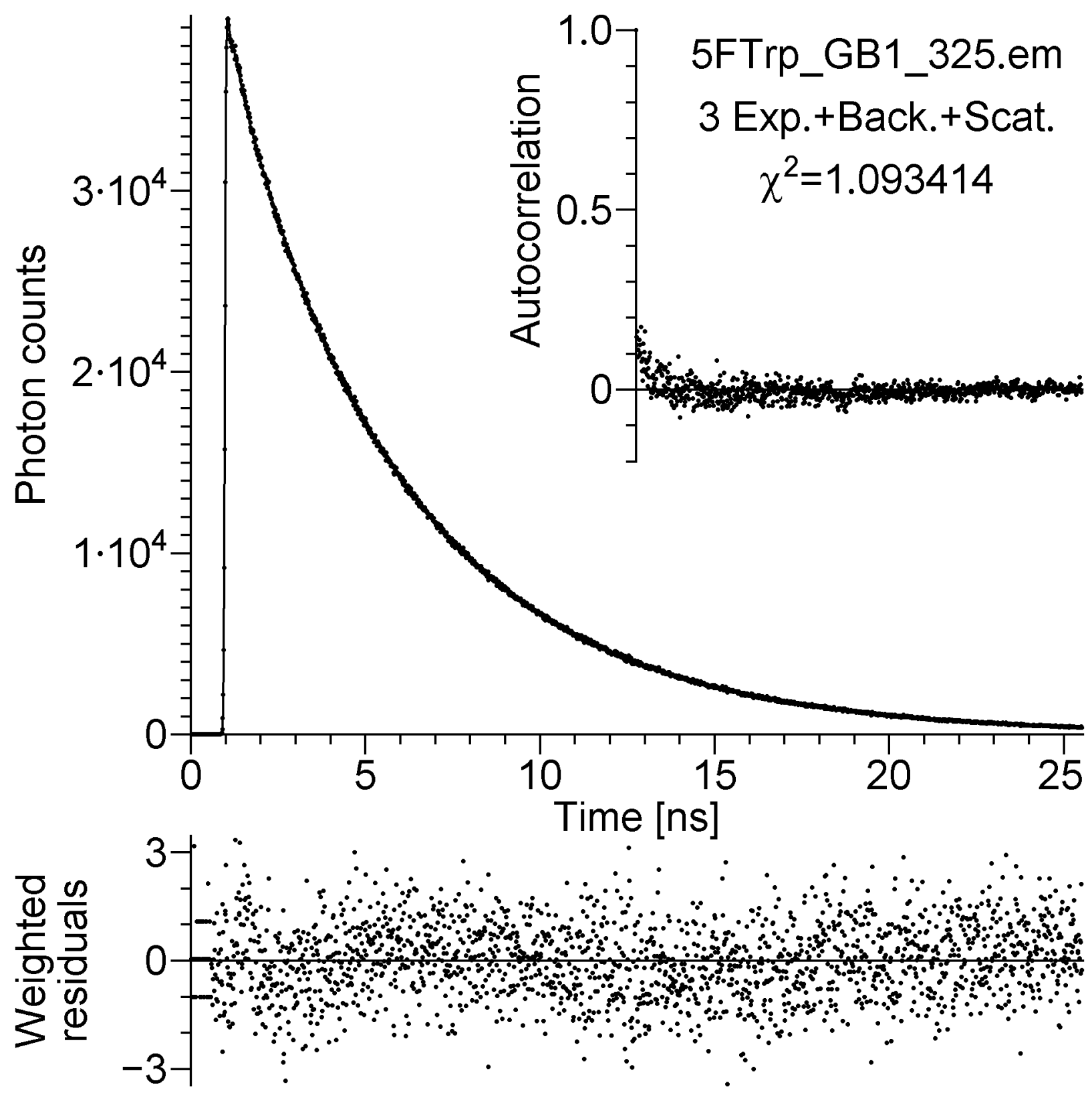
Figure 7S. Emission of 5FTrp GB1 at $325 \mathrm{~nm}$, fit by the quadruple-exponential model.
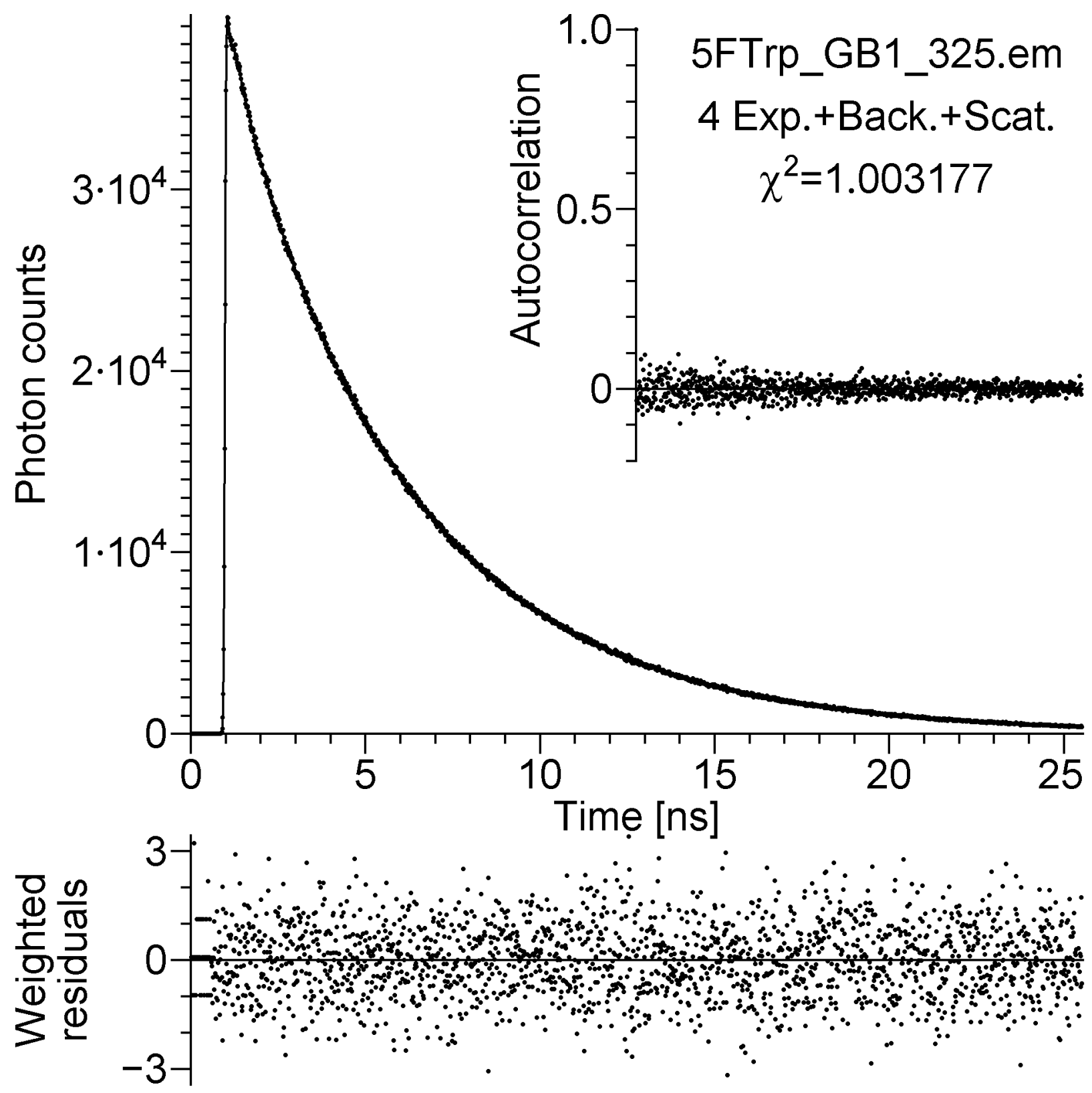
Figure 8S. Emission of 5FTrp GB1 at 325nm, fit by the quintuple-exponential model.
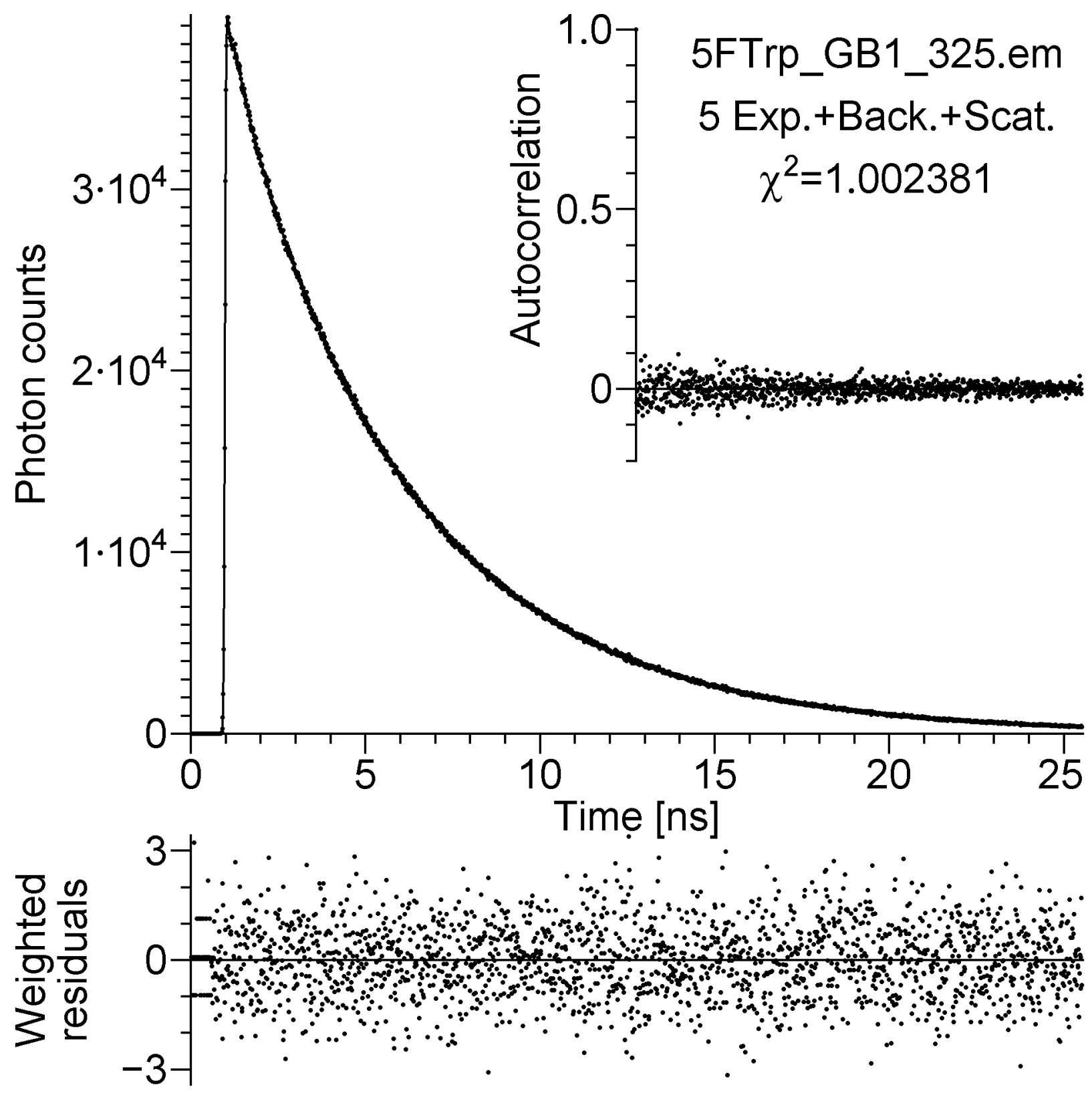
Figure 9S. Emission of 5FTrp GB1 at 355nm, fit by the monoexponential model.
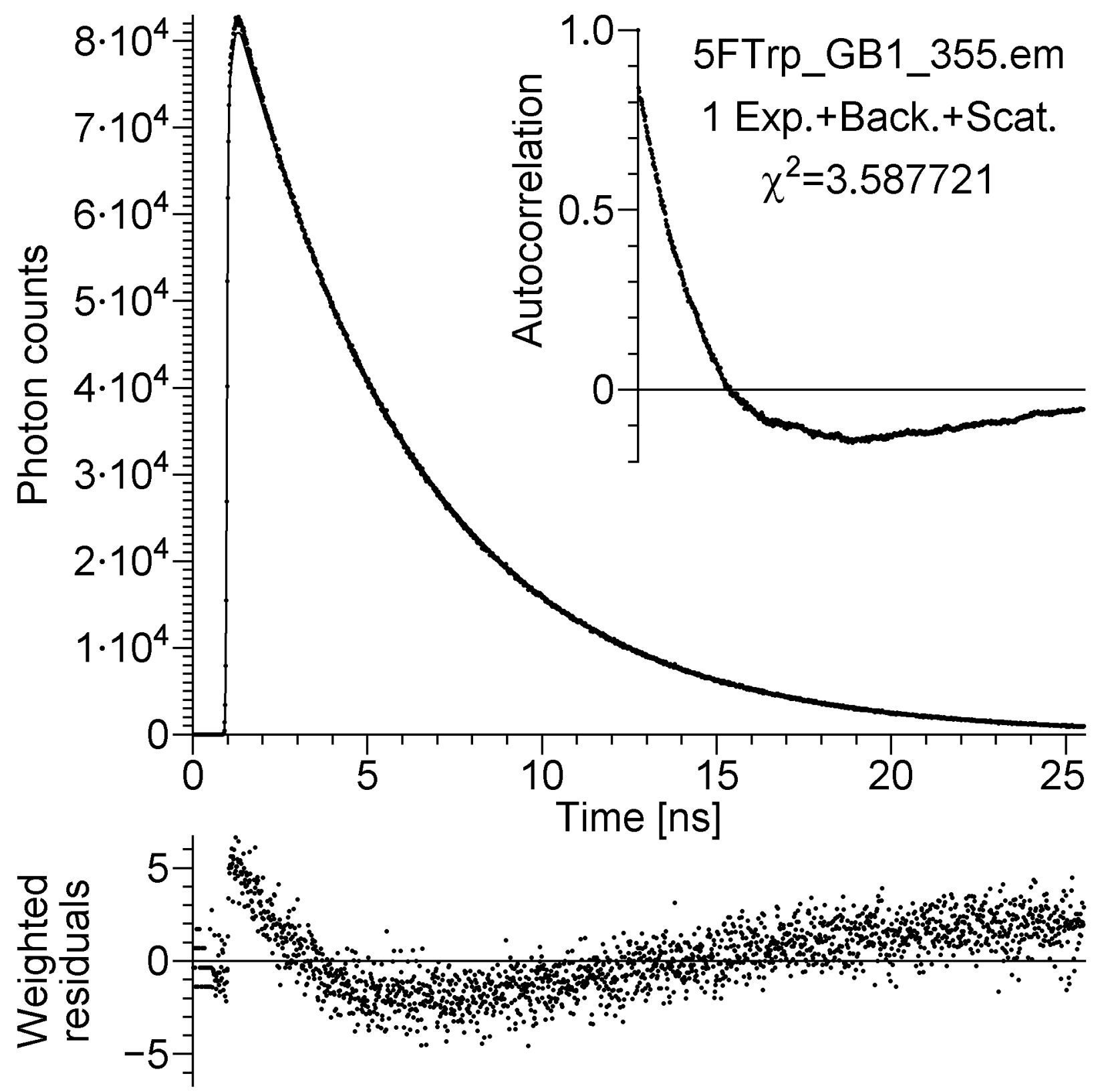
Figure 10S. Emission of 5FTrp GB1 at $355 \mathrm{~nm}$, fit by the double-exponential model.

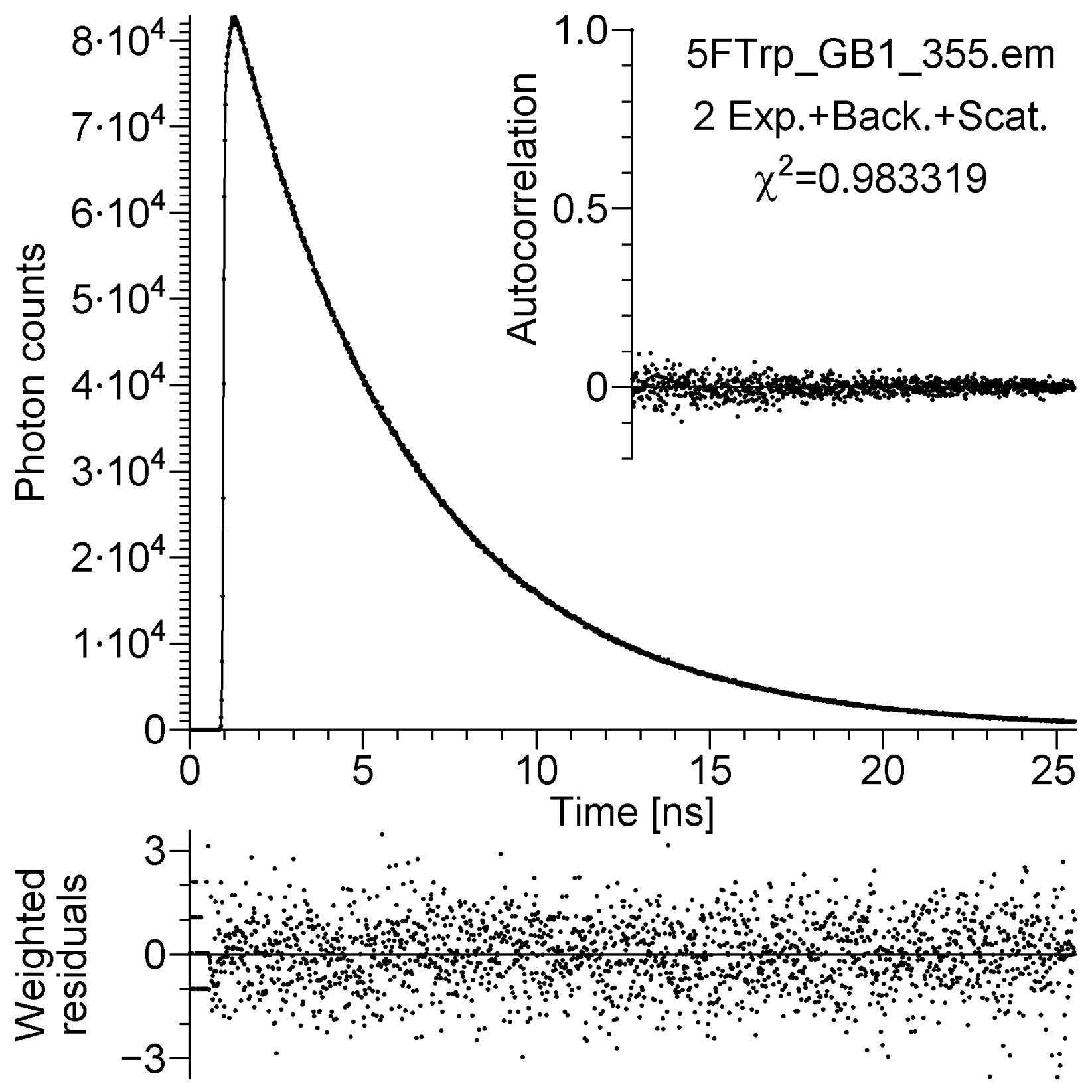


Figure 11S. Emission of 5FTrp GB1 at 355nm, fit by the triple-exponential model.

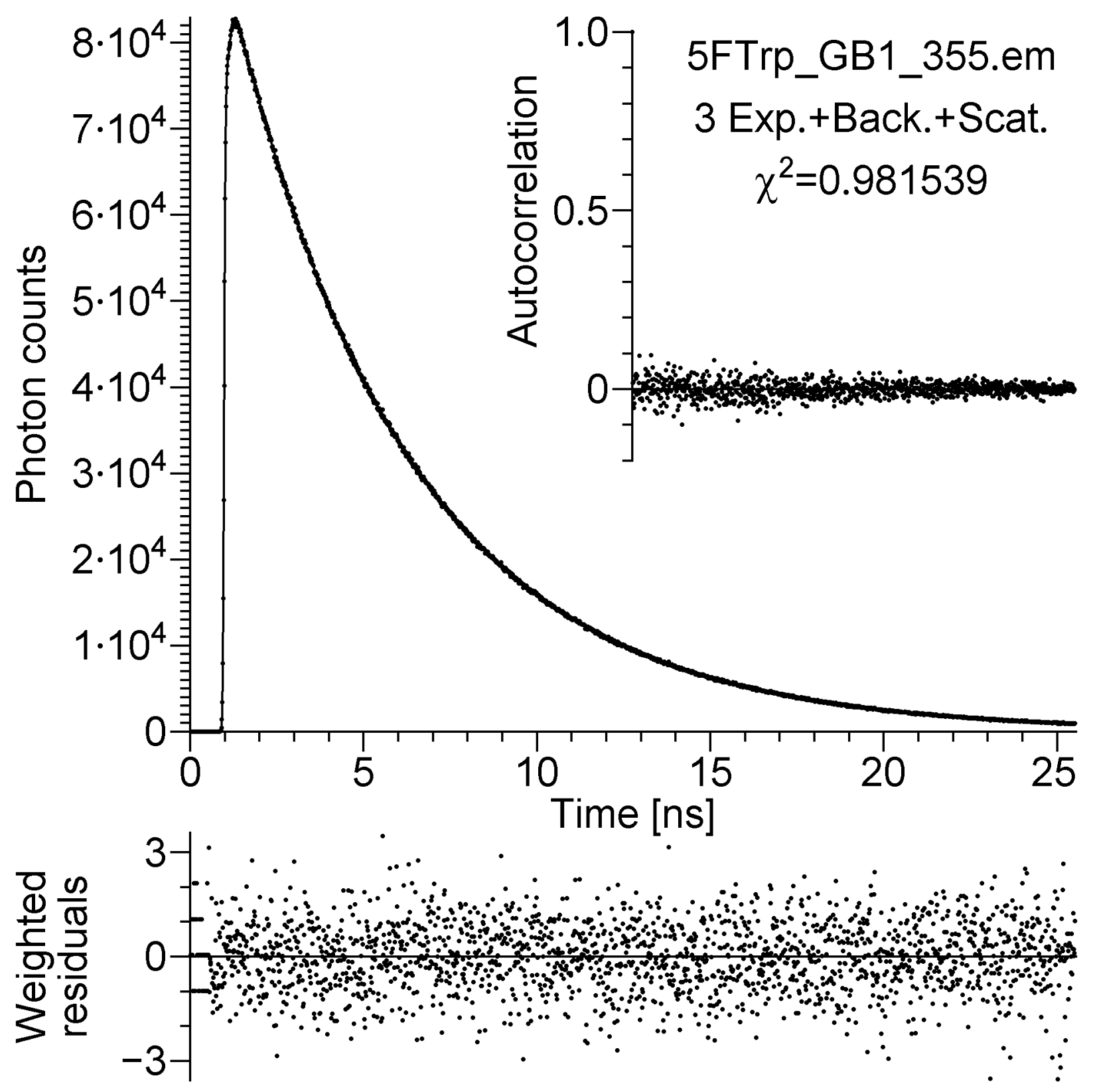


Figure 12S. Emission of 5FTrp GB1 at 355nm, fit by the quadruple-exponential model.

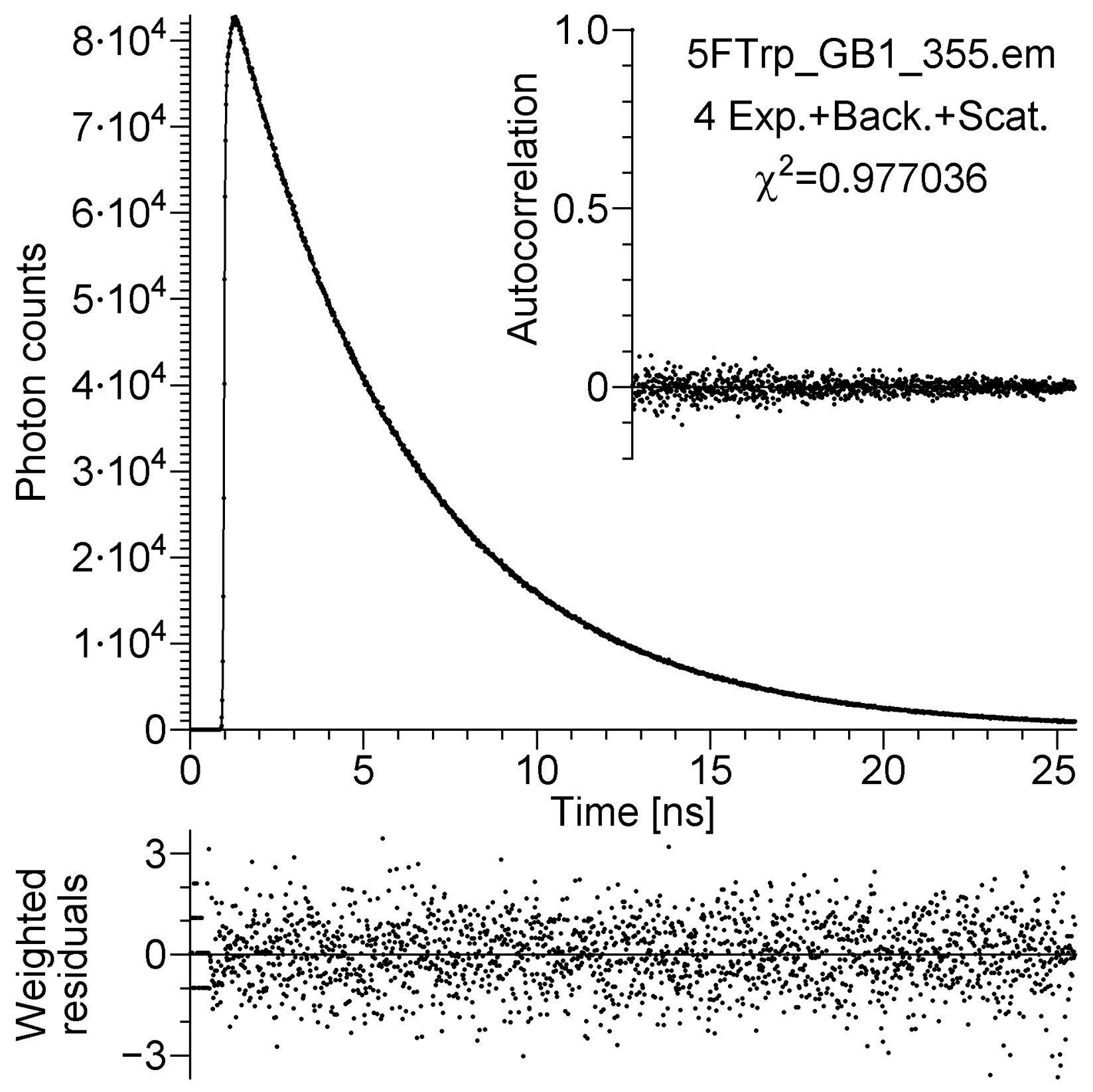


Figure 13S. Emission of 5FTrp GB1 at 355nm, fit by the quintuple-exponential model.

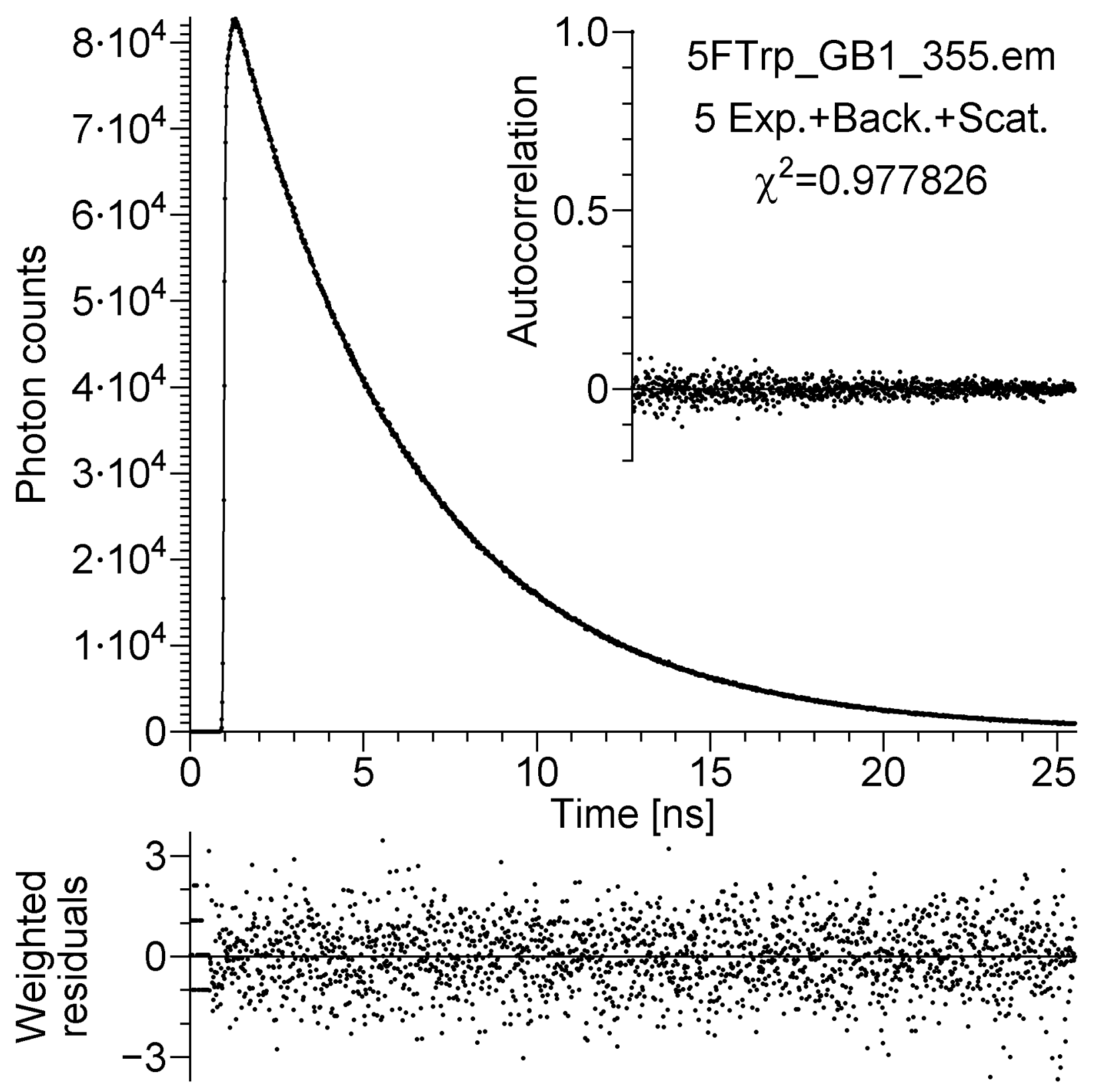


Figure 14S. Emission of 5FTrp GB1 at 390nm, fit by the monoexponential model.
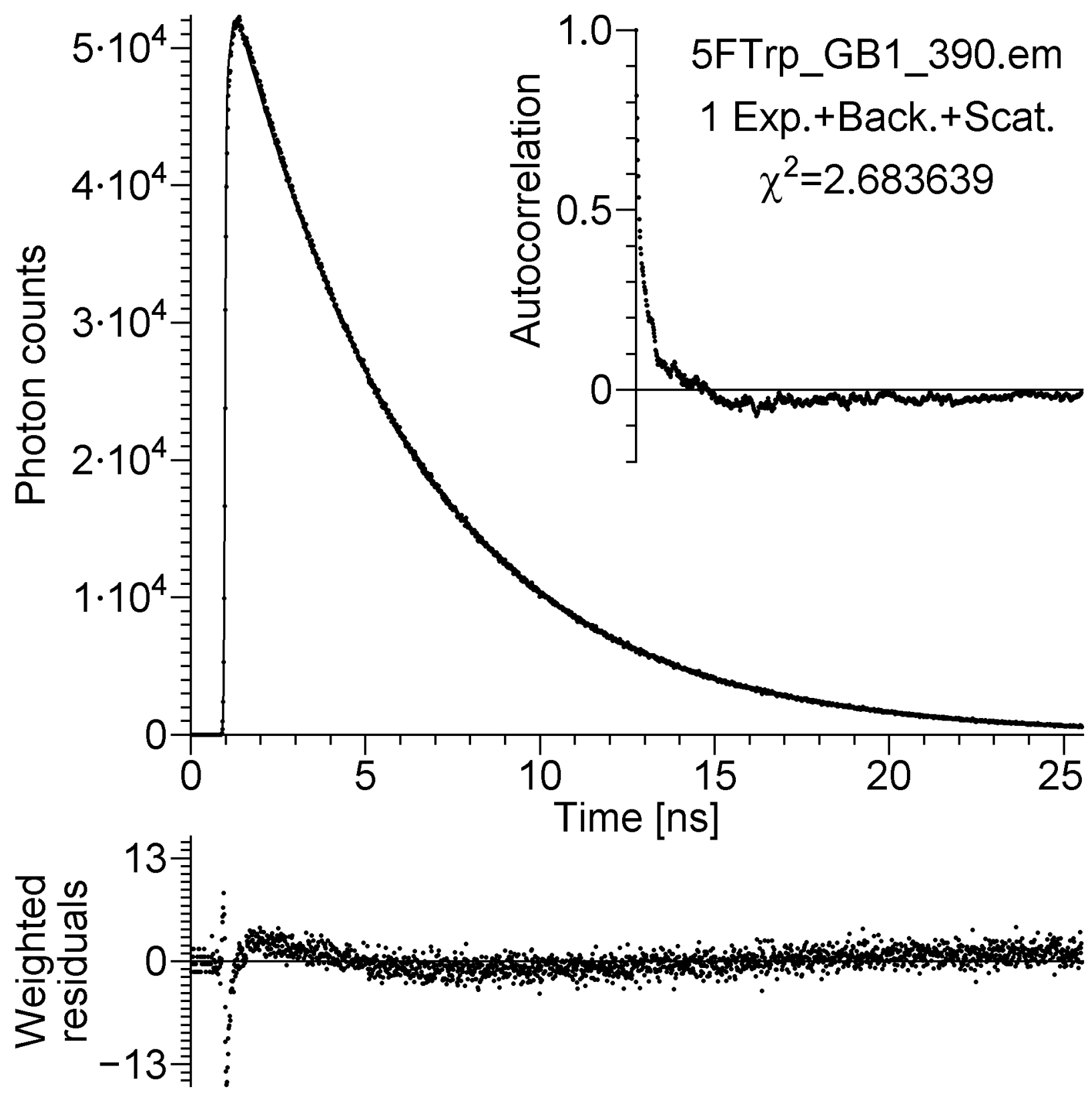
Figure 15S. Emission of 5FTrp GB1 at 390nm, fit by the double-exponential model.

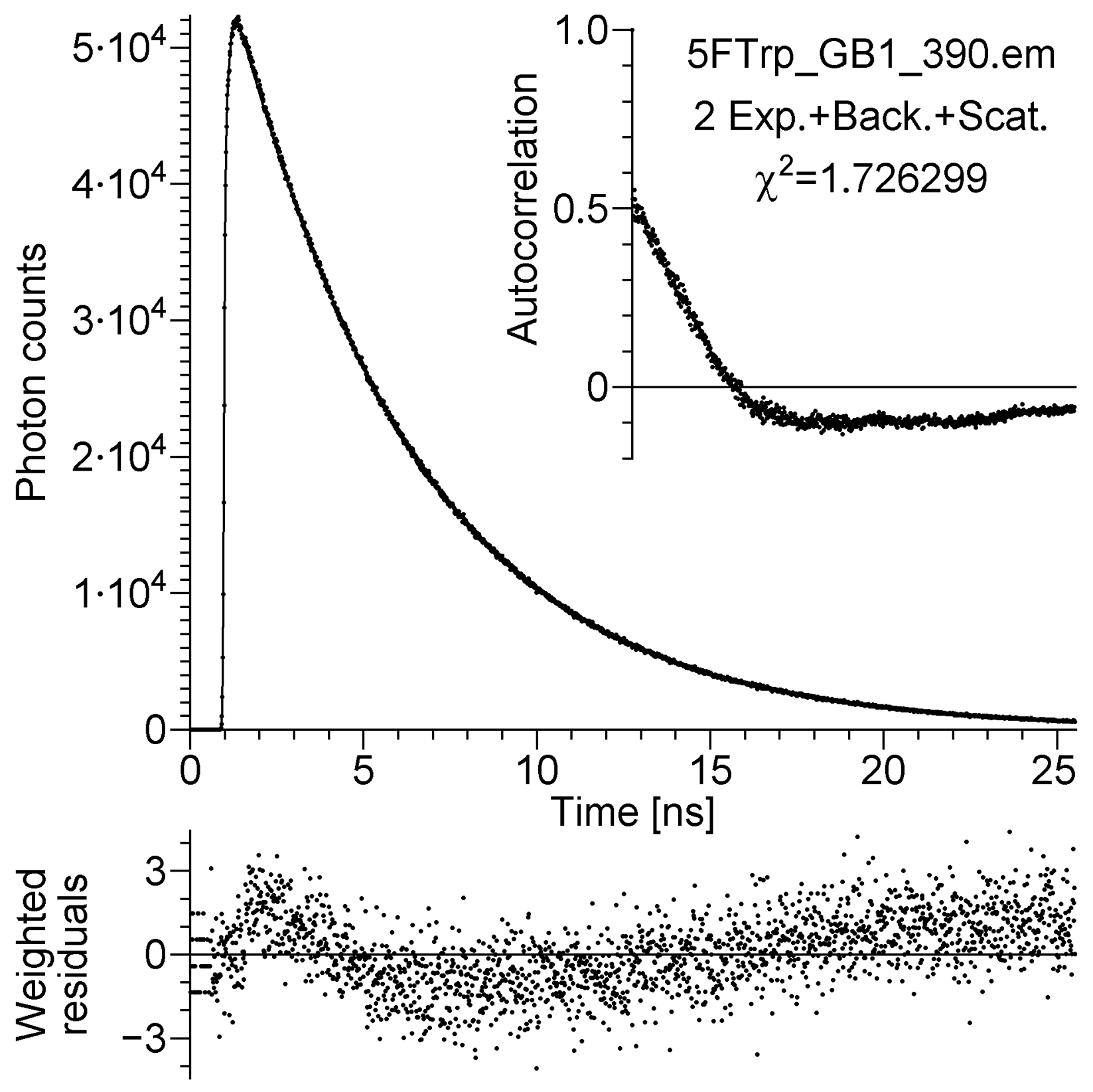


Figure 16S. Emission of 5FTrp GB1 at 390nm, fit by the triple-exponential model.

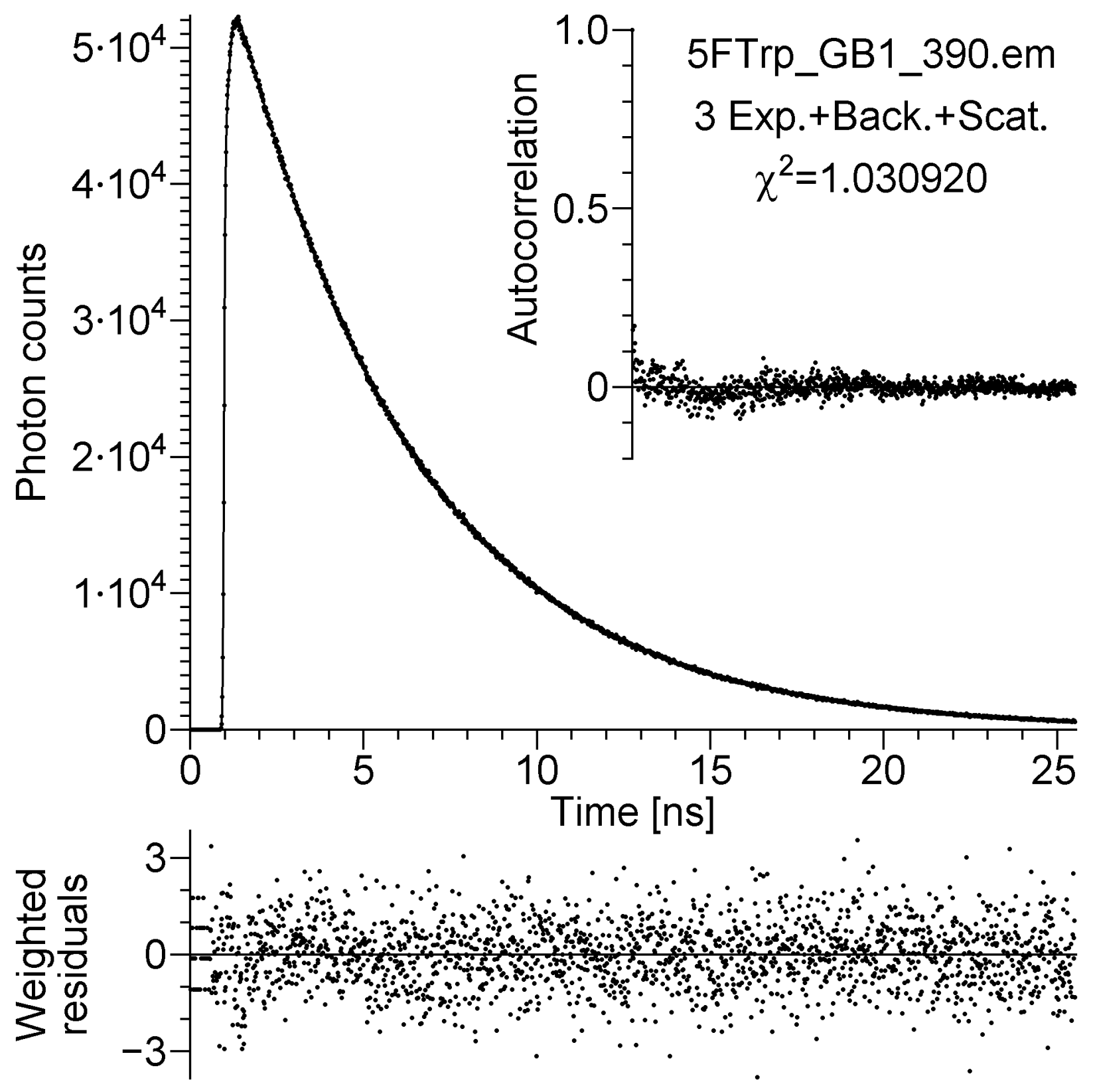


Figure 17S. Emission of 5FTrp GB1 at 390nm, fit by the quadruple-exponential model.

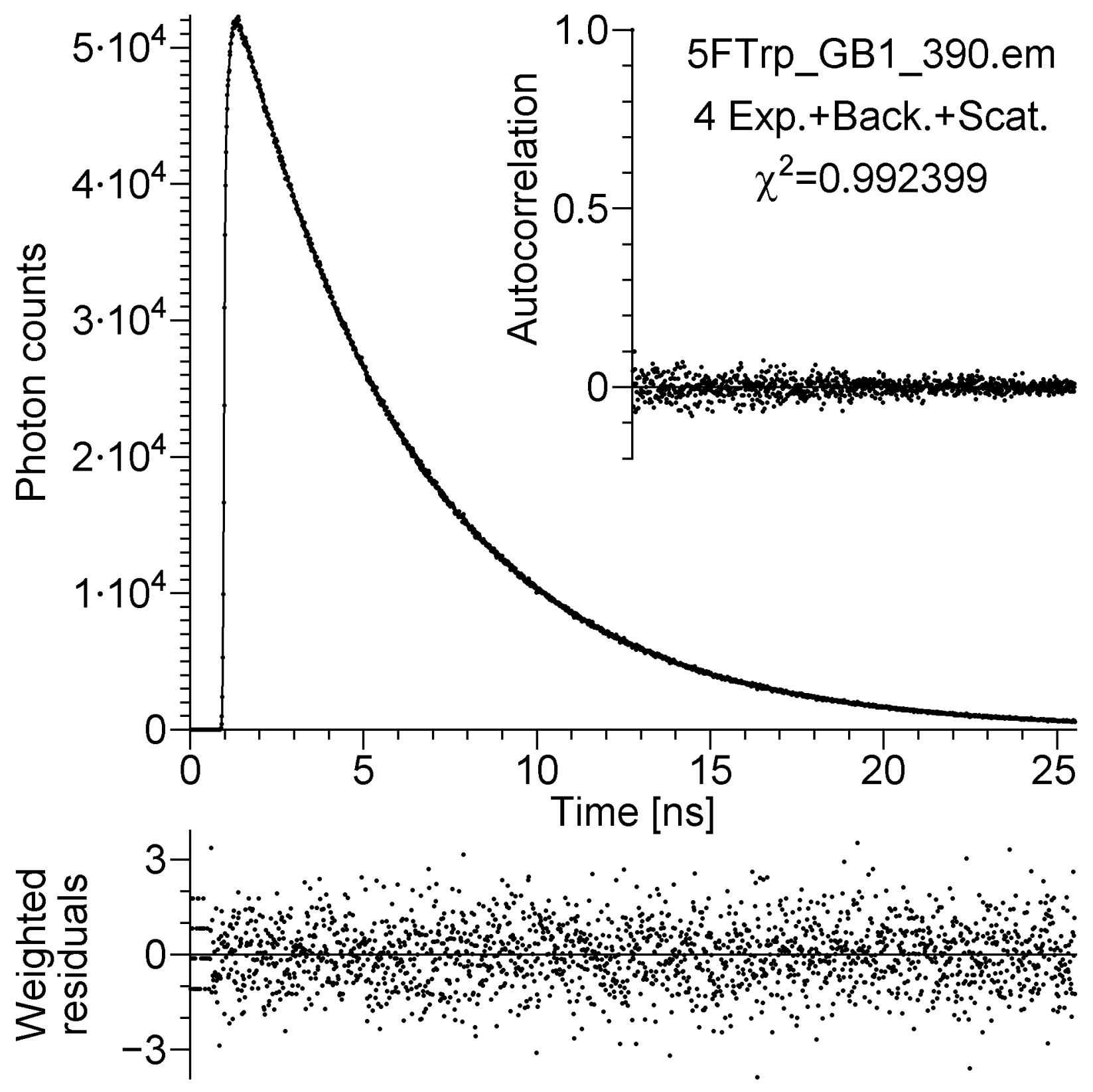


Figure 18S. Emission of 5FTrp GB1 at 390nm, fit by the quintuple-exponential model.

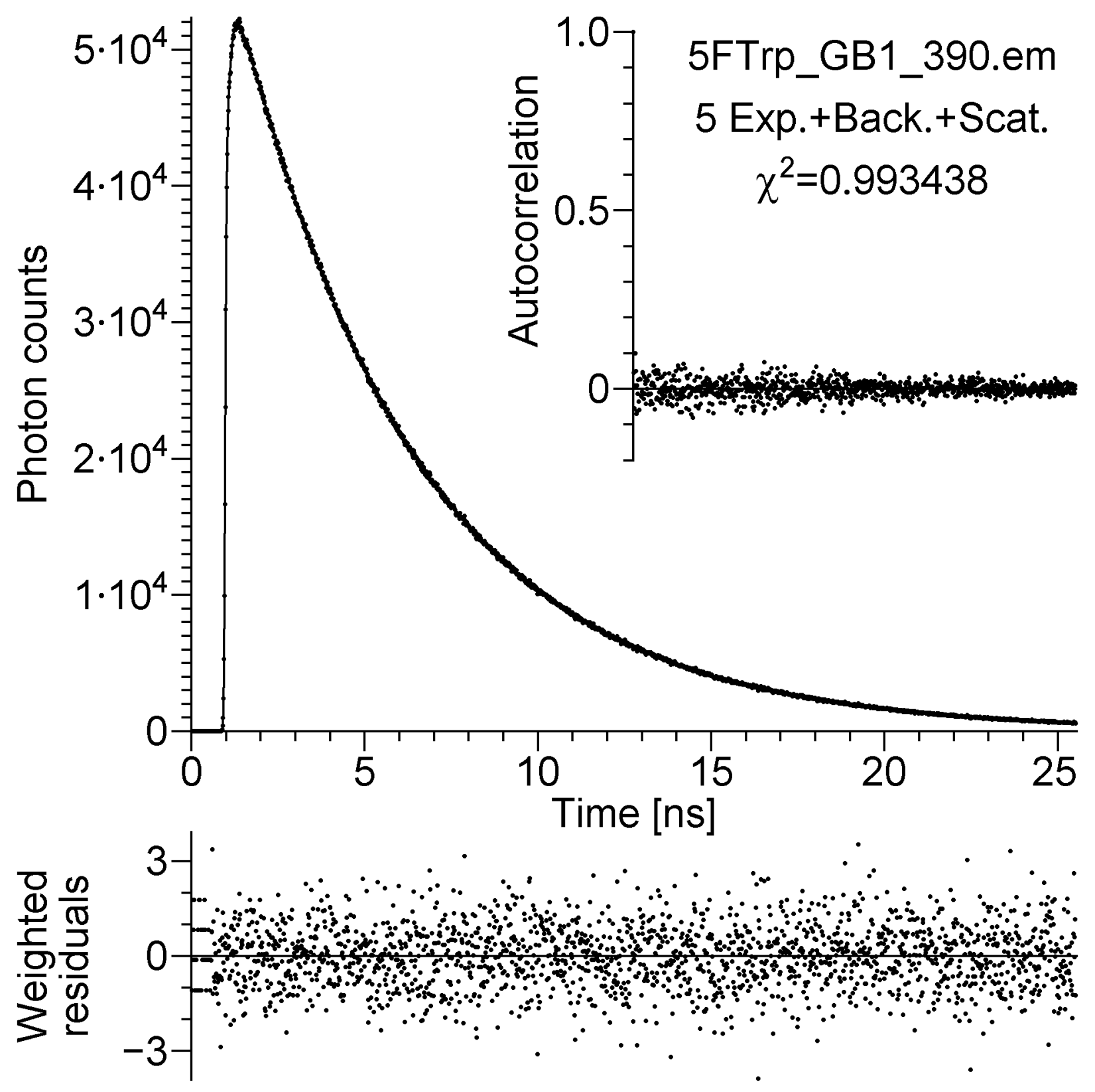


Figure 19S. Emission of unmodified GB1 at 320nm, fit by the monoexponential model.

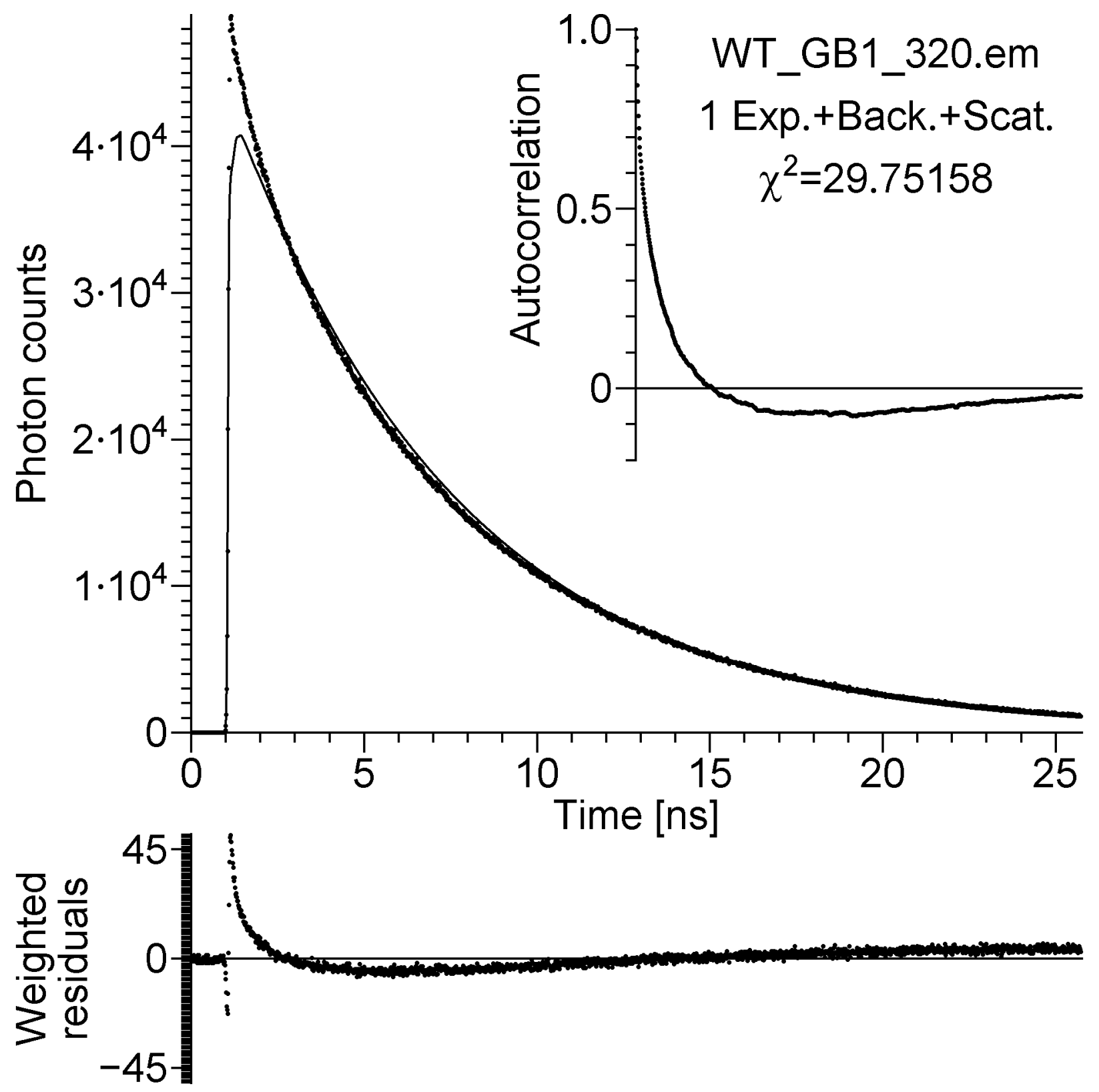


Figure 20S. Emission of unmodified GB1 at 320nm, fit by the double-exponential model.

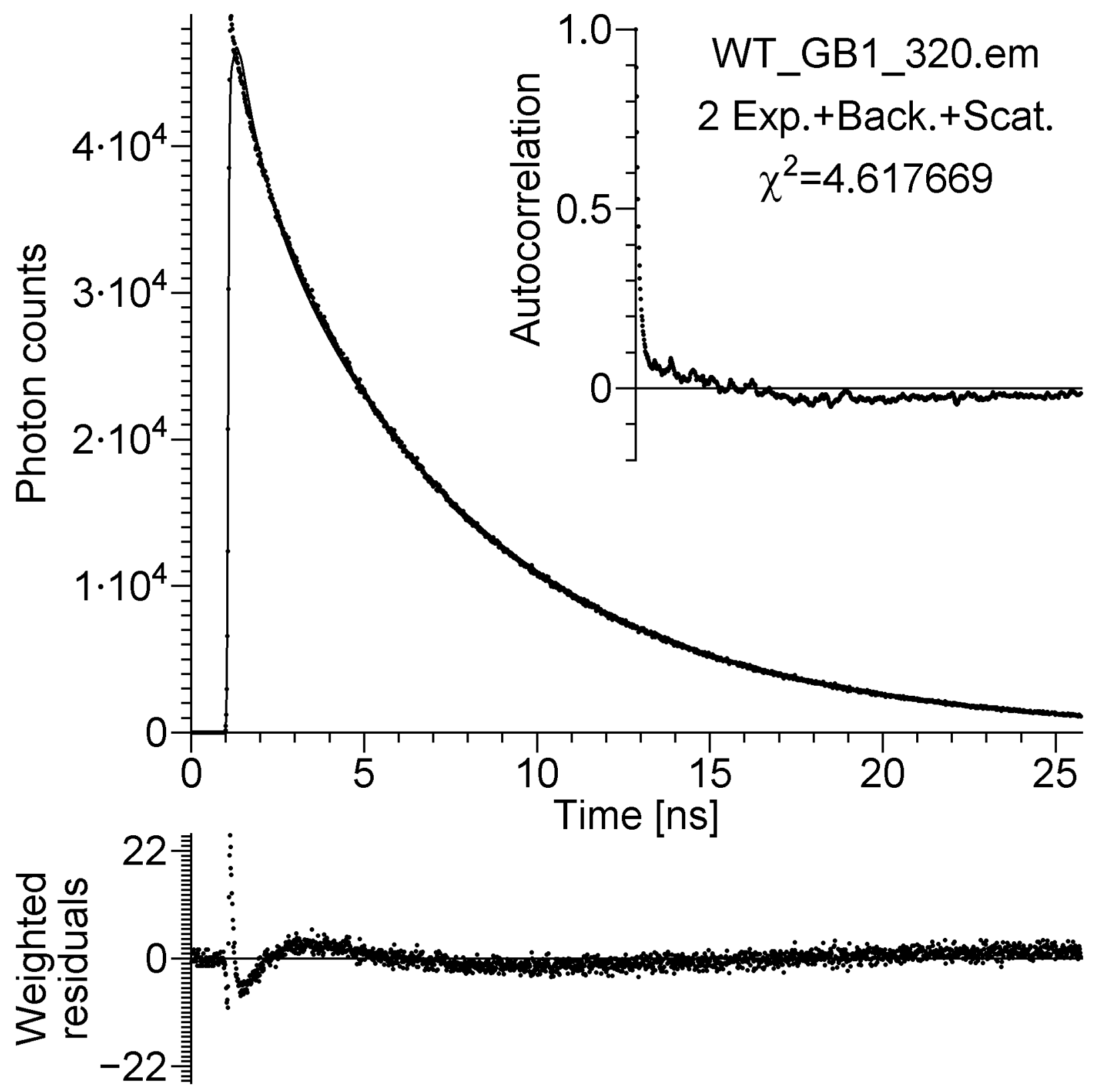


Figure 21S. Emission of unmodified GB1 at $320 \mathrm{~nm}$, fit by the triple-exponential model.

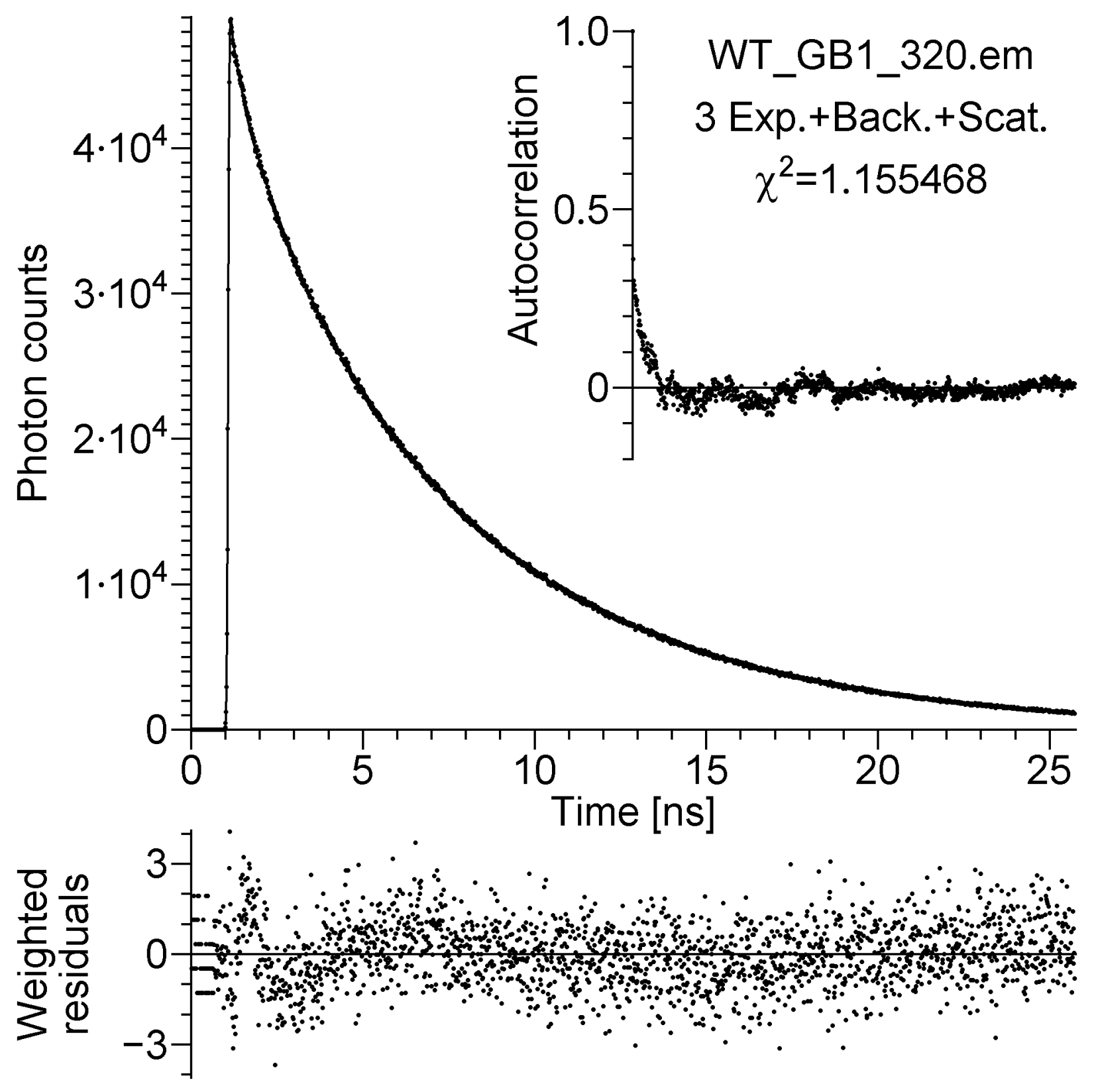


Figure 22S. Emission of unmodified GB1 at $320 \mathrm{~nm}$, fit by the quadruple-exponential model.

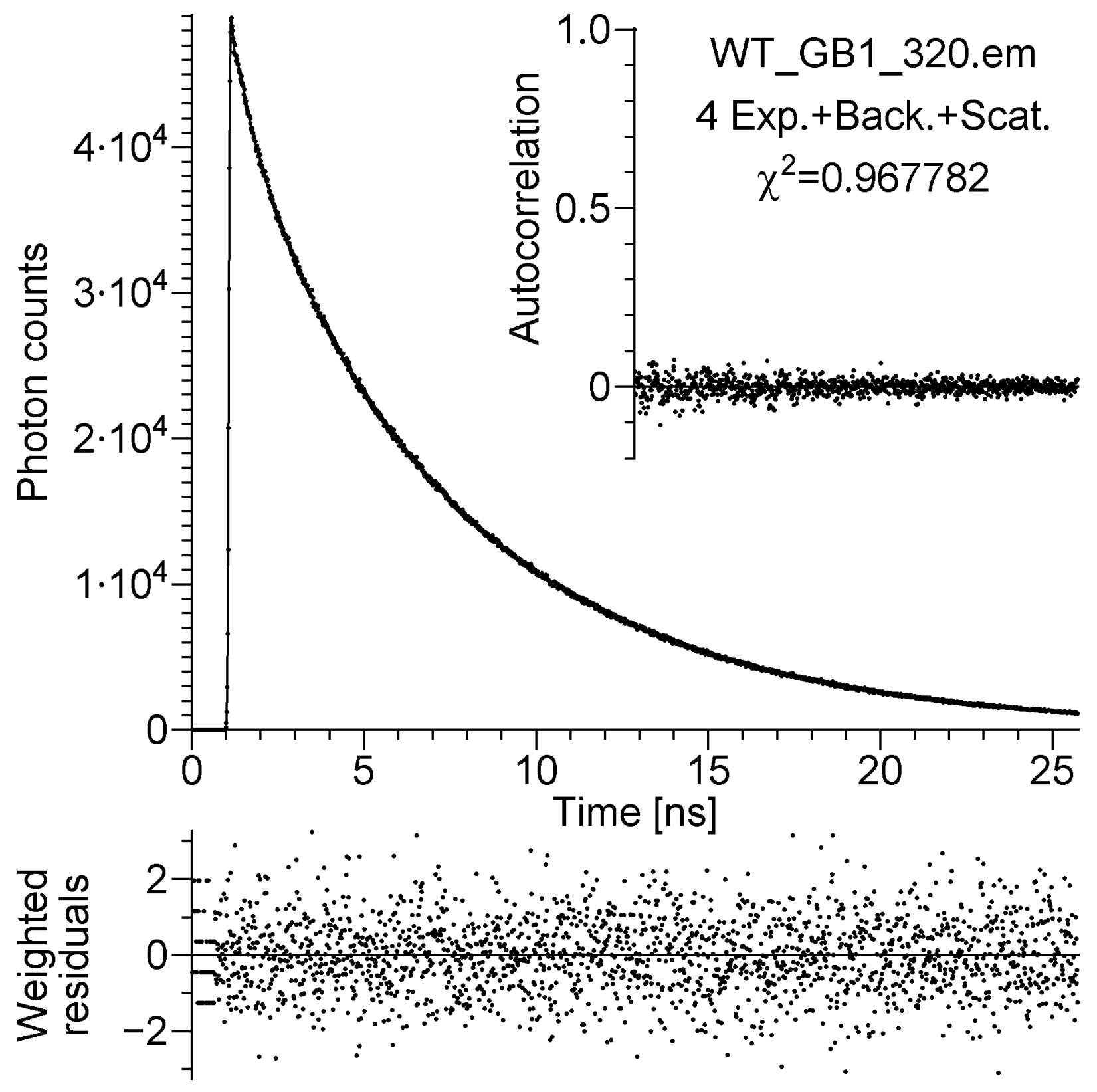


Figure 23S. Emission of unmodified GB1 at 320nm, fit by the quintuple-exponential model.

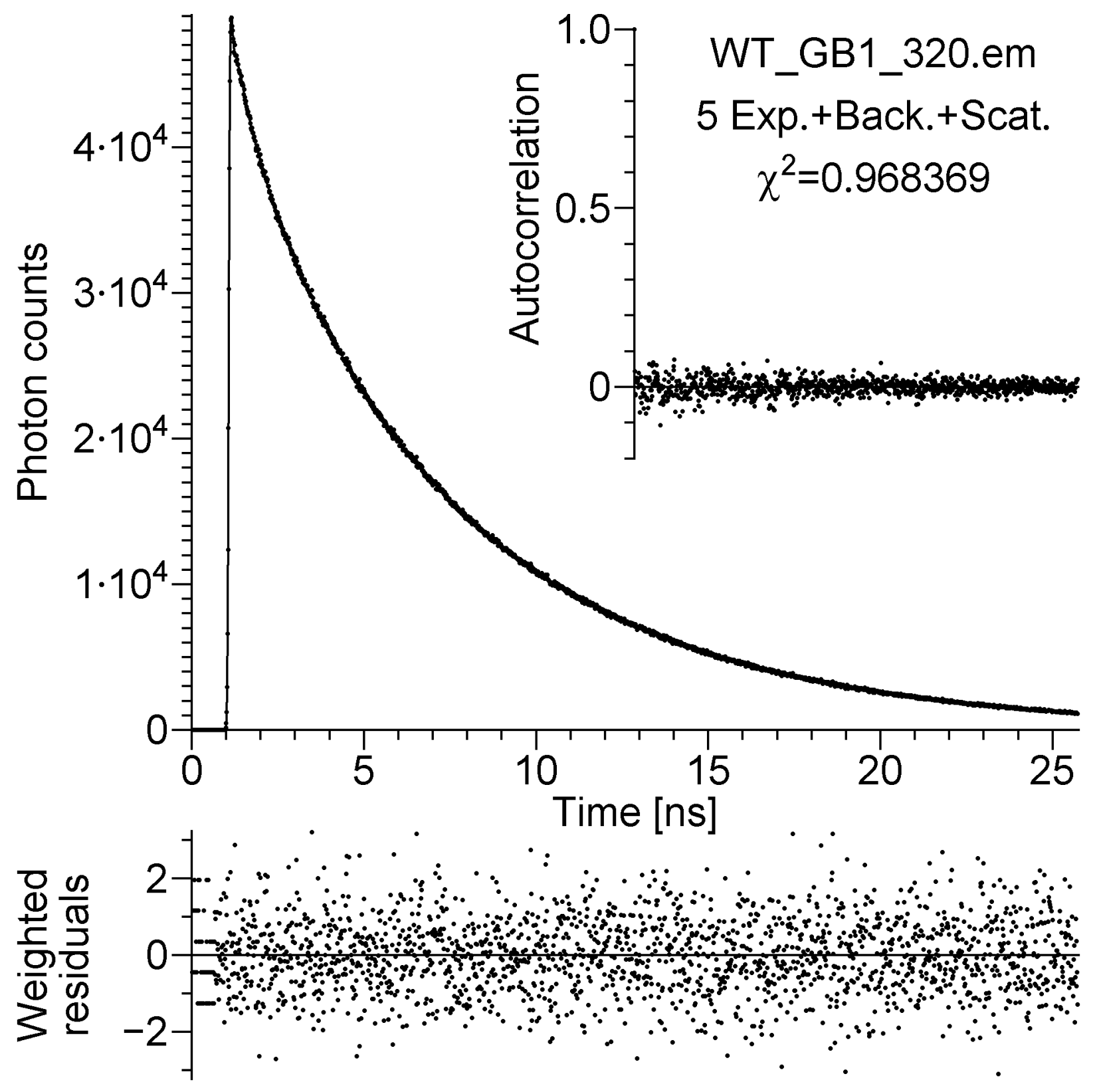


Figure 24S. Emission of unmodified GB1 at 350nm, fit by the monoexponential model.

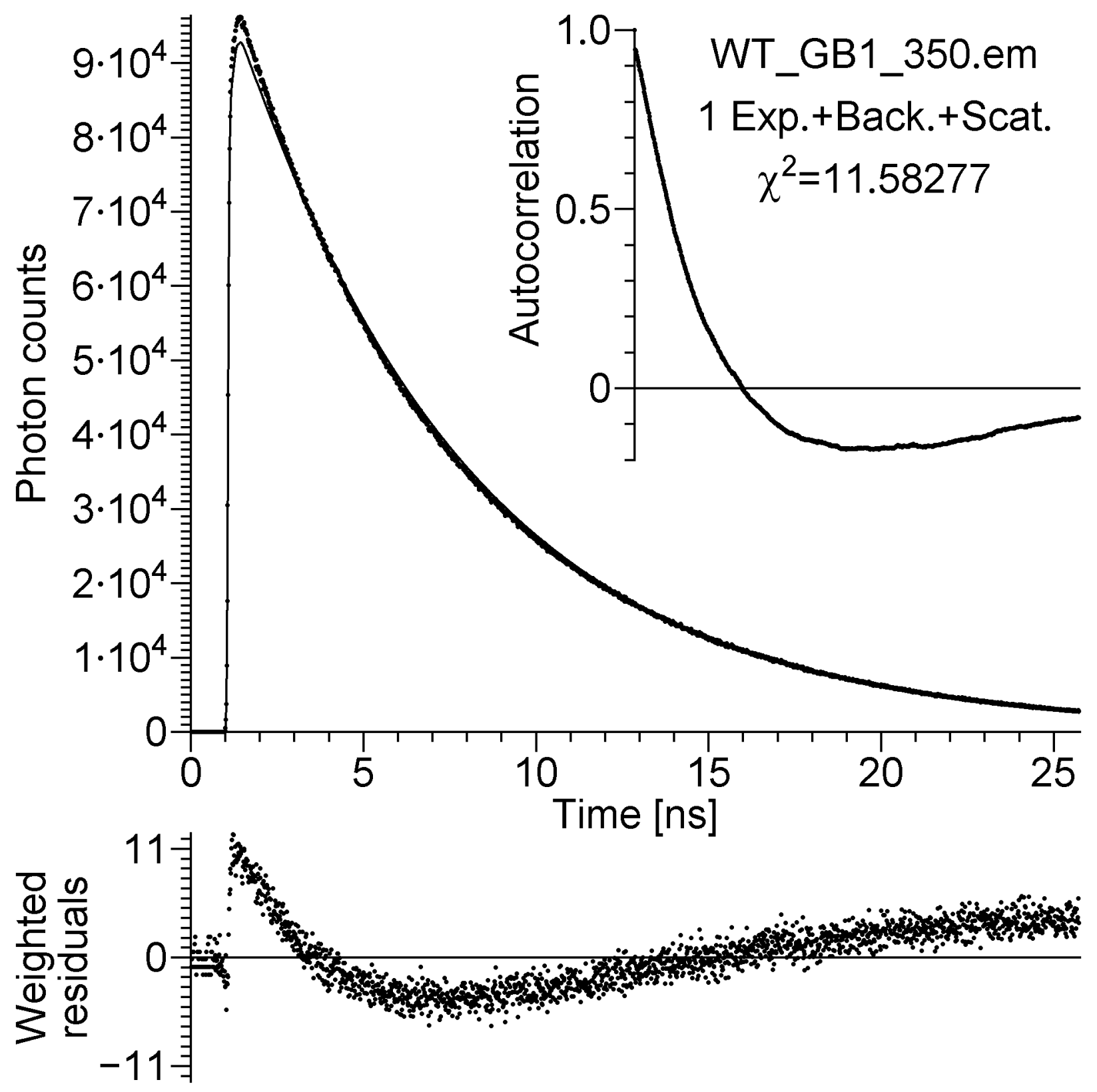


Figure 25S. Emission of unmodified GB1 at 350nm, fit by the double-exponential model.

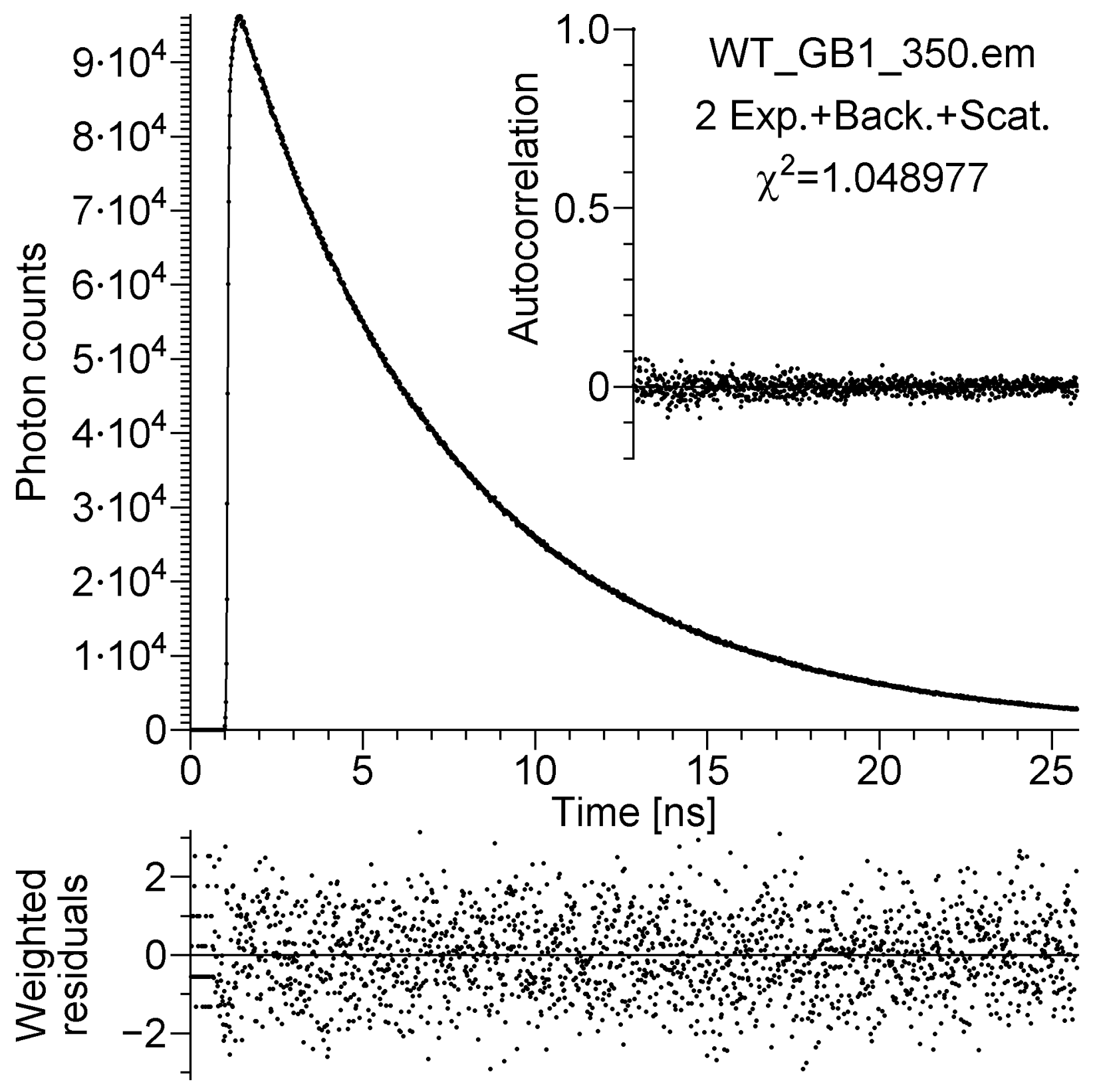


Figure 26S. Emission of unmodified GB1 at 350nm, fit by the triple-exponential model.

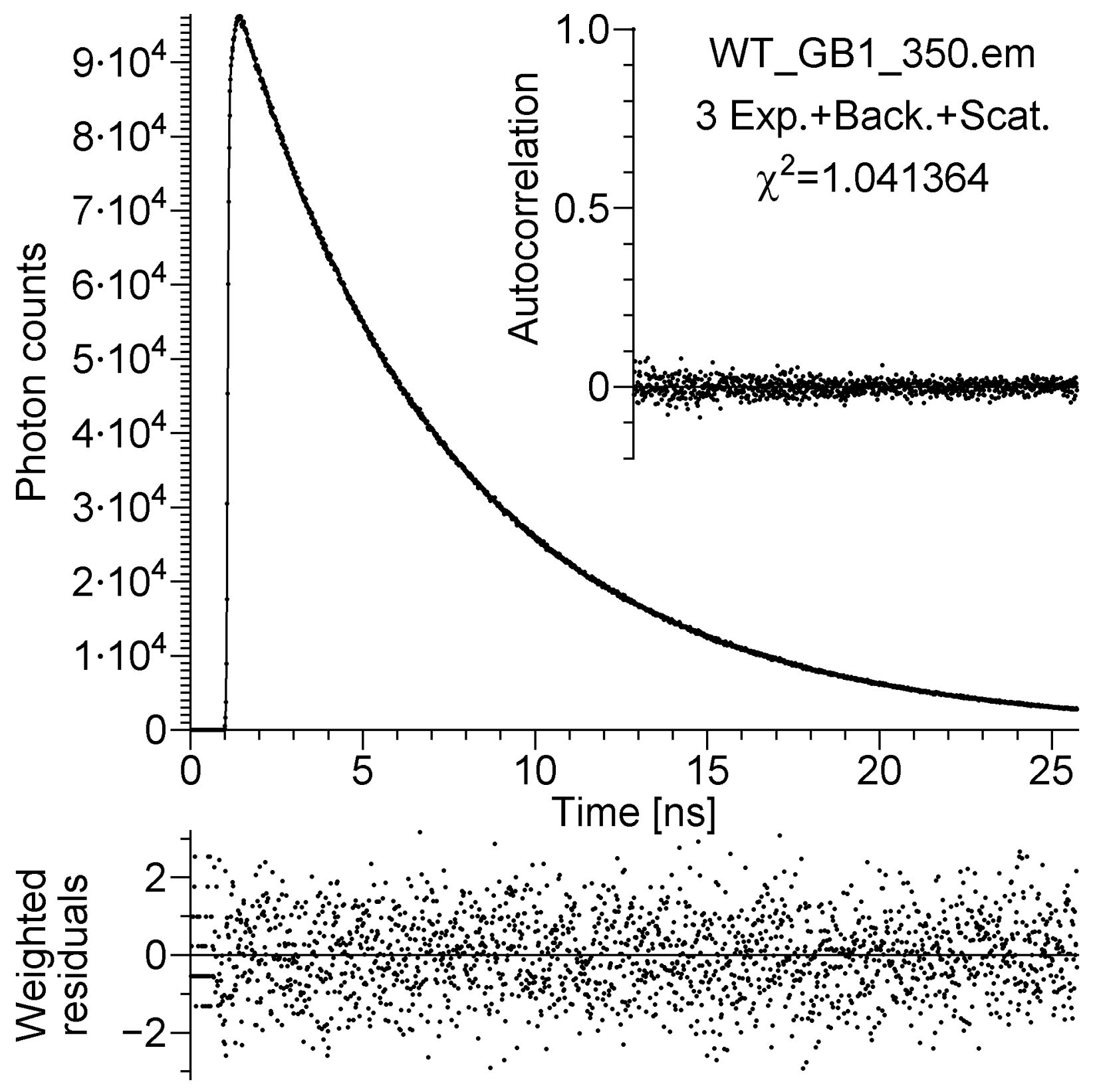


Figure 27S. Emission of unmodified GB1 at 350nm, fit by the quadruple-exponential model.

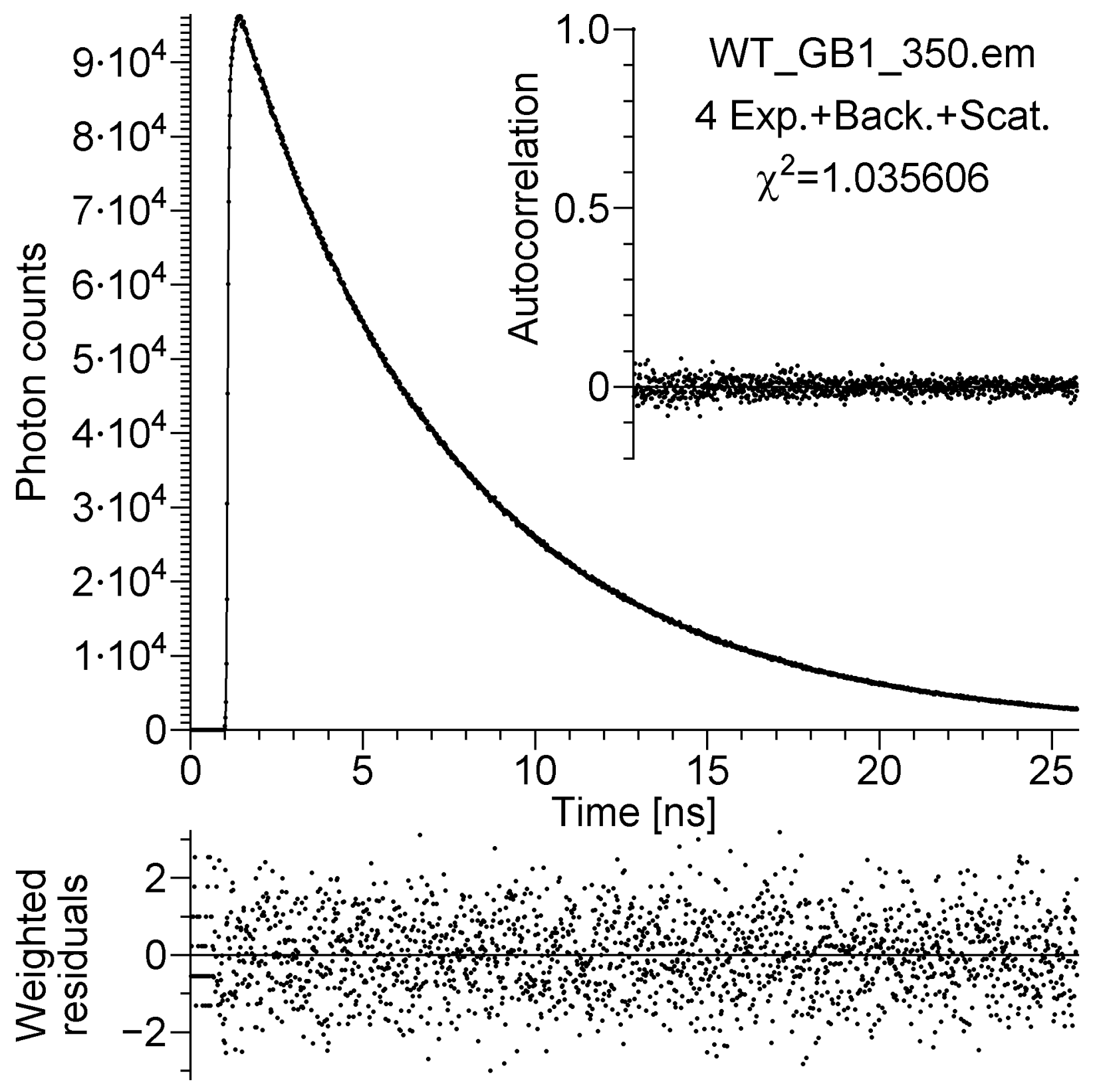


Figure 28S. Emission of unmodified GB1 at 350nm, fit by the quintuple-exponential model.
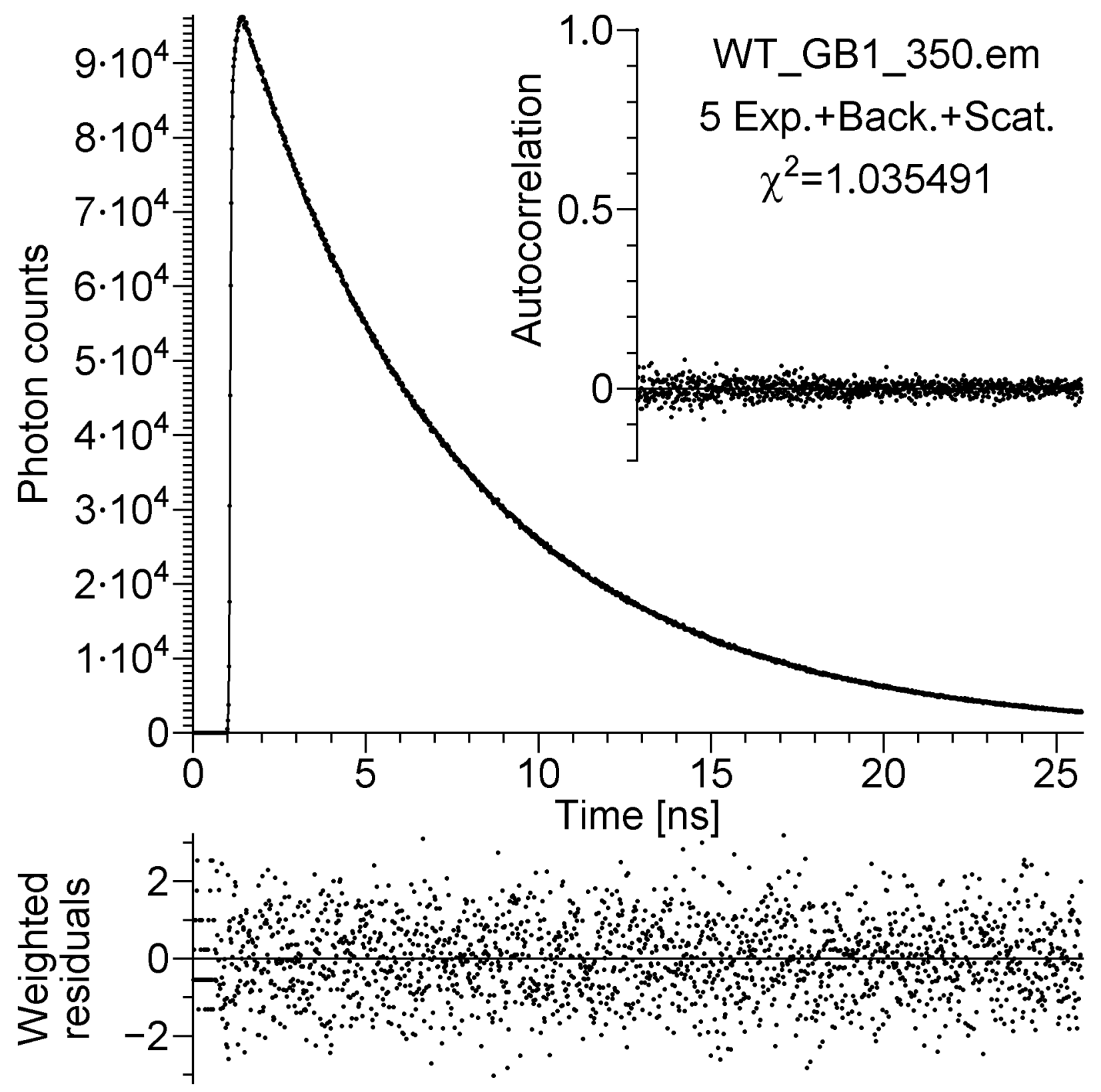
Figure 29S. Emission of unmodified GB1 at $385 \mathrm{~nm}$, fit by the monoexponential model.

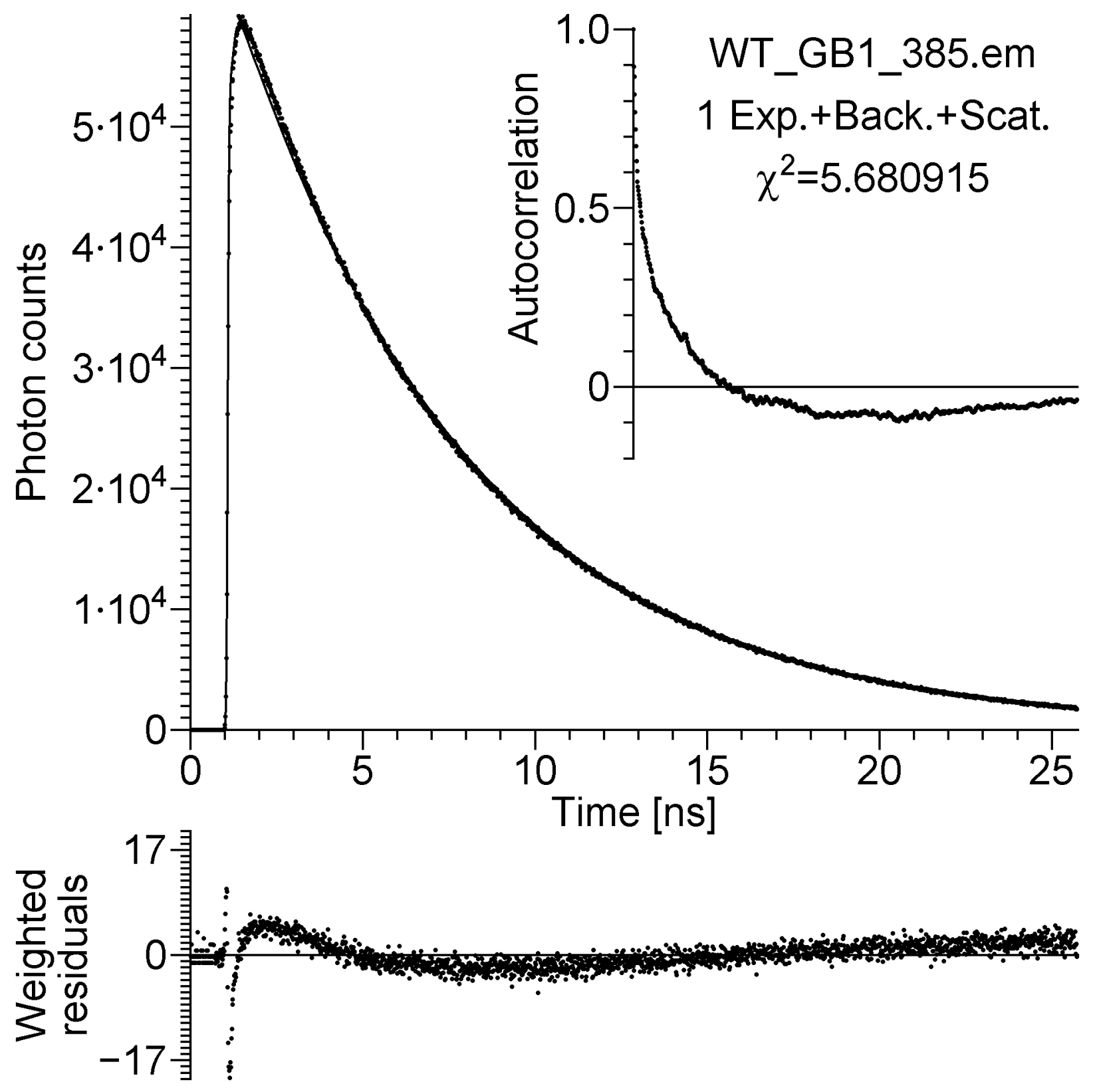


Figure 30S. Emission of unmodified GB1 at 385nm, fit by the double-exponential model.
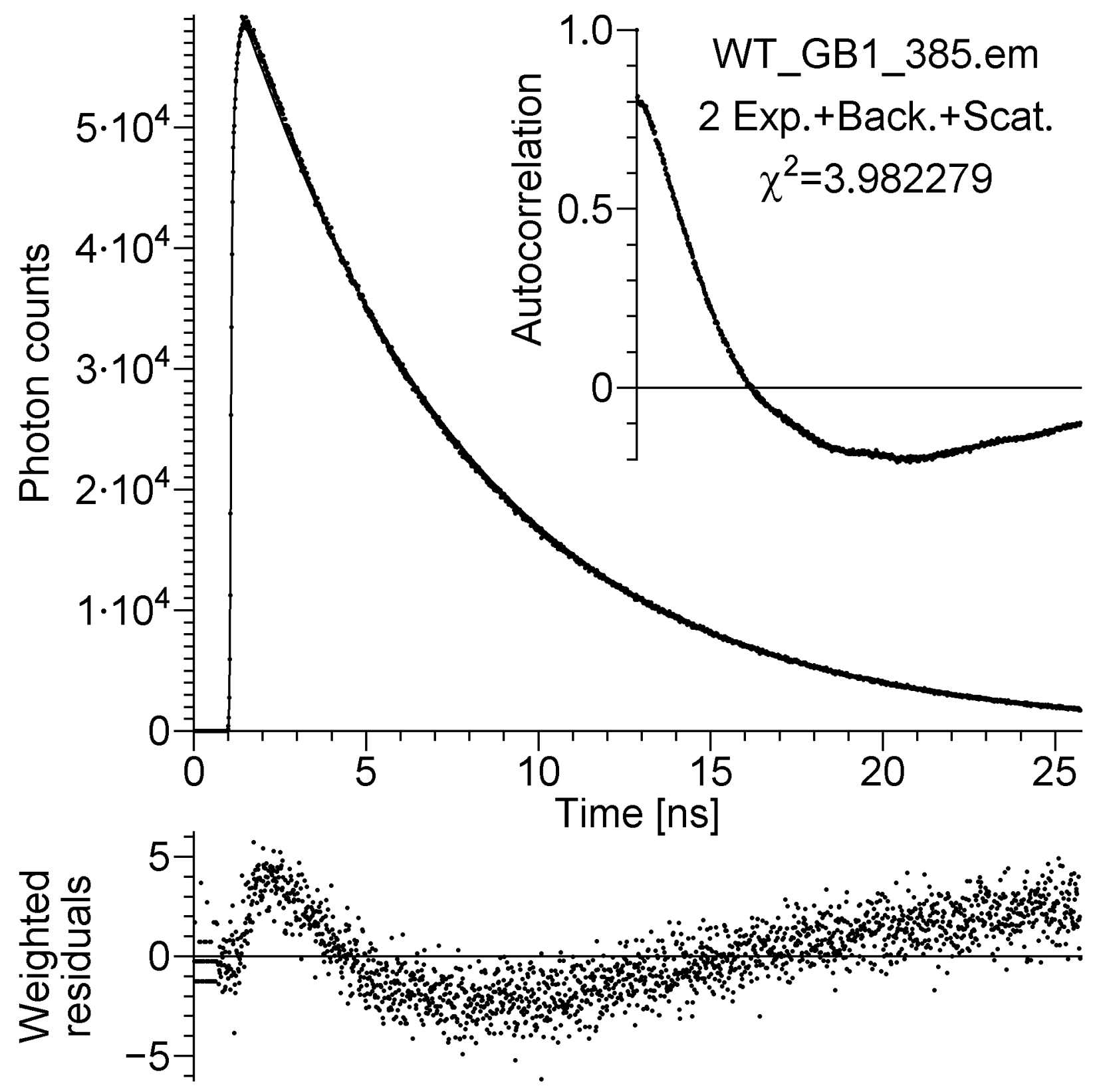
Figure 31S. Emission of unmodified GB1 at $385 \mathrm{~nm}$, fit by the triple-exponential model.

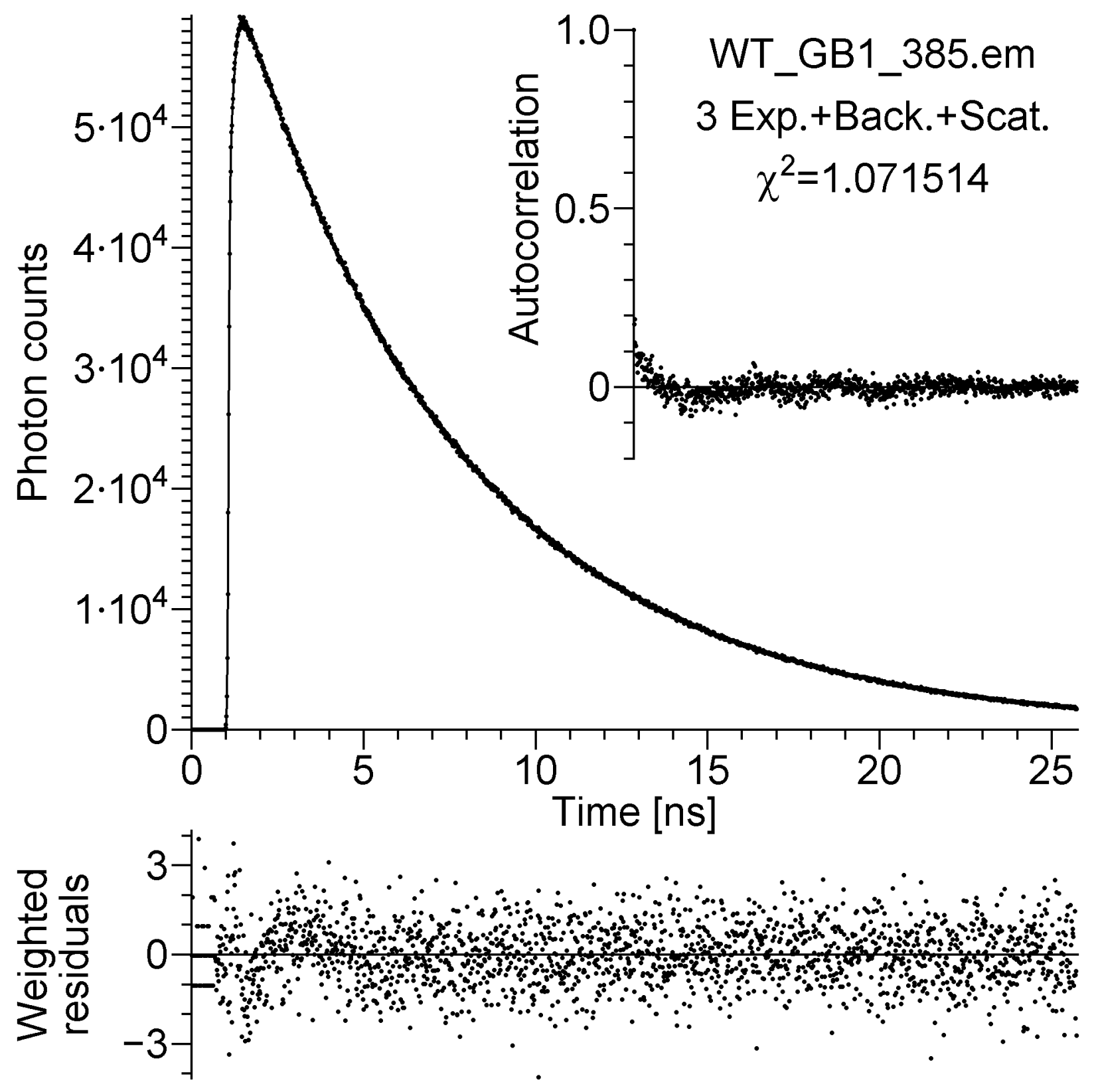


Figure 32S. Emission of unmodified GB1 at $385 \mathrm{~nm}$, fit by the quadruple-exponential model.

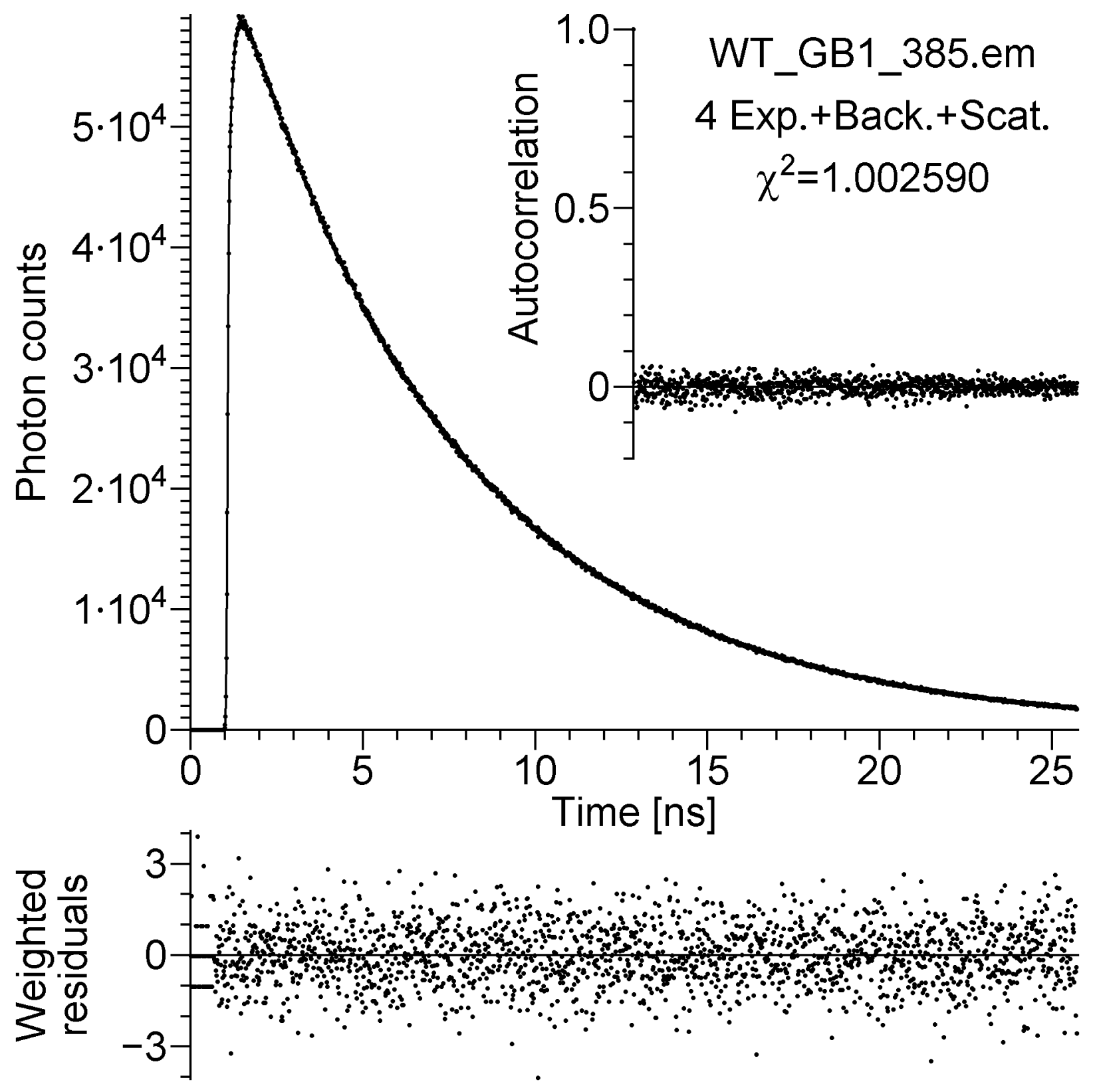


Figure 33S. Emission of unmodified GB1 at 385nm, fit by the quintuple-exponential model.

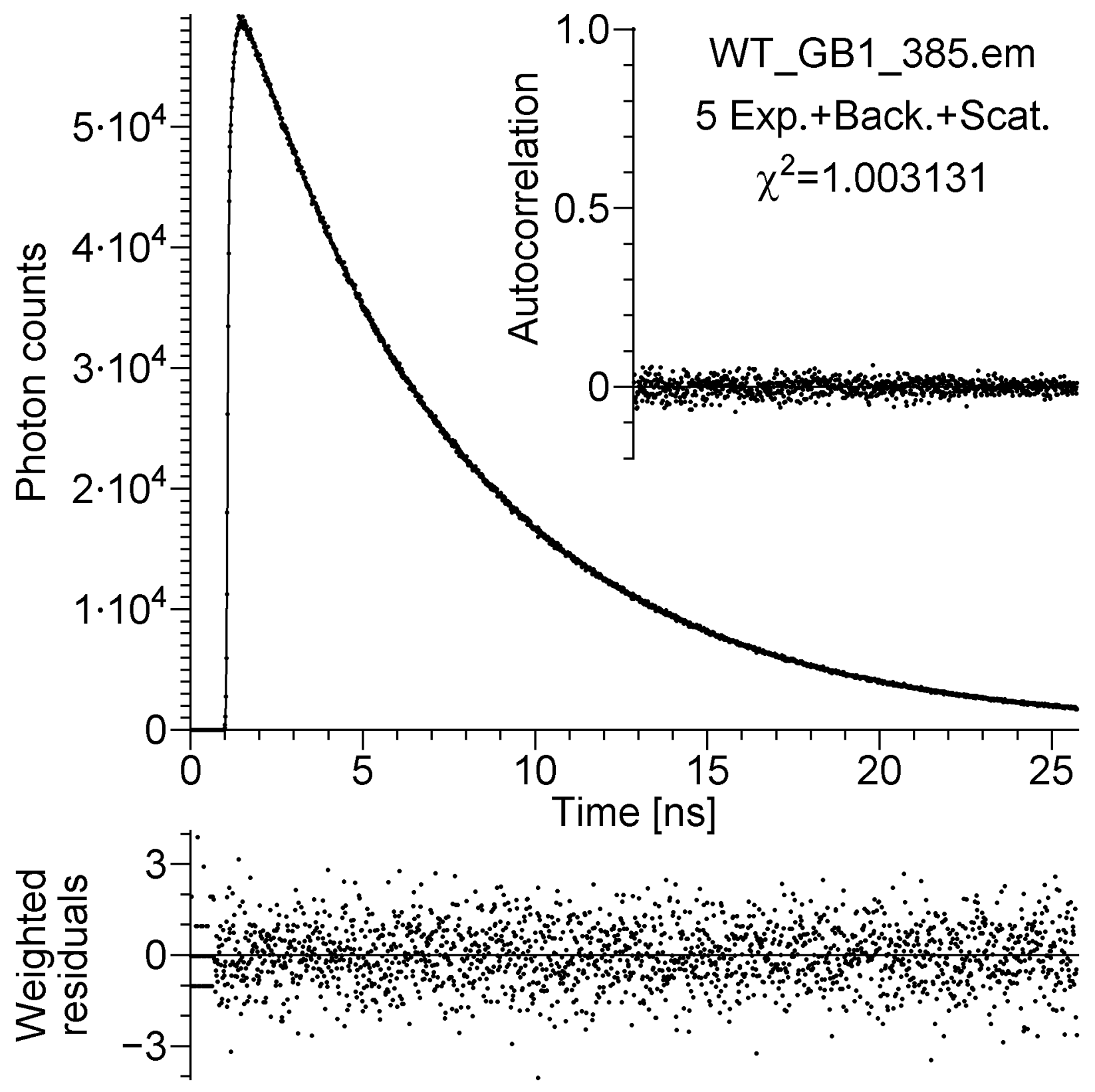


Table 1S. Best-fit parameters for 5FTrp GB1 emission at 325nm, quadruple-exponential model. $\sigma \alpha_{n}$ and $\sigma \tau_{n}$ represent standard deviations for $\alpha_{n}$ and $\tau_{n}$.

\begin{tabular}{|c|r|r|c|c|}
\hline$n$ & \multicolumn{1}{|c|}{$\alpha_{n}$} & $\sigma \alpha_{n}$ & $\tau_{n}$ & $\sigma \tau_{n}$ \\
\hline unit & \multicolumn{2}{|c|}{ photon/channel } & \multicolumn{2}{|c|}{ ns } \\
\hline 1 & 14802 & 497 & 0.073 & 0.004 \\
2 & 3227 & 161 & 0.687 & 0.066 \\
3 & 8267 & 1955 & 3.657 & 0.306 \\
4 & 29237 & 2109 & 5.629 & 0.069 \\
\hline
\end{tabular}

Table 2S. Best-fit parameters for 5FTrp GB1 emission at 355nm, double-exponential model. $\sigma \alpha_{n}$ and $\sigma \tau_{n}$ represent standard deviations for $\alpha_{n}$ and $\tau_{n}$.

\begin{tabular}{|c|c|c|c|c|}
\hline$n$ & $\alpha_{n}$ & $\sigma \alpha_{n}$ & $\tau_{n}$ & $\sigma \tau_{n}$ \\
\hline unit & \multicolumn{2}{|c|}{ photon/channel } & \multicolumn{2}{|c|}{ ns } \\
\hline 1 & 11022 & 623 & 3.045 & 0.077 \\
2 & 78052 & 644 & 5.508 & 0.011 \\
\hline
\end{tabular}

Table 3S. Best-fit parameters for 5FTrp GB1 emission at 390nm, triple-exponential model. $\sigma \alpha_{n}$ and $\sigma \tau_{n}$ represent standard deviations for $\alpha_{n}$ and $\tau_{n}$.

\begin{tabular}{|c|c|r|c|c|}
\hline$n$ & $\alpha_{n}$ & $\sigma \alpha_{n}$ & $\tau_{n}$ & $\sigma \tau_{n}$ \\
\hline unit & \multicolumn{2}{|c|}{ photon/channel } & \multicolumn{2}{|c|}{ ns } \\
\hline 1 & -8343 & 296 & 0.118 & 0.005 \\
2 & 24666 & 7047 & 4.514 & 0.195 \\
3 & 32334 & 7074 & 5.865 & 0.151 \\
\hline
\end{tabular}


Table 4S. Best-fit parameters for unmodified Trp GB1 emission at 320nm, quadrupleexponential model. $\sigma \alpha_{n}$ and $\sigma \tau_{n}$ represent standard deviations for $\alpha_{n}$ and $\tau_{n}$.

\begin{tabular}{|c|c|c|c|c|}
\hline$n$ & $\alpha_{n}$ & $\sigma \alpha_{n}$ & $\tau_{n}$ & $\sigma \tau_{n}$ \\
\hline unit & \multicolumn{2}{|c|}{ photon/channel } & \multicolumn{2}{|c|}{ ns } \\
\hline 1 & 20841 & 521 & 0.076 & 0.003 \\
2 & 5490 & 207 & 0.618 & 0.044 \\
3 & 7607 & 205 & 3.032 & 0.135 \\
4 & 36384 & 342 & 7.128 & 0.021 \\
\hline
\end{tabular}

Table 5S. Best-fit parameters for unmodified Trp GB1 emission at 350nm, doubleexponential model. $\sigma \alpha_{n}$ and $\sigma \tau_{n}$ represent standard deviations for $\alpha_{n}$ and $\tau_{n}$.

\begin{tabular}{|c|c|c|c|c|}
\hline$n$ & $\alpha_{n}$ & $\sigma \alpha_{n}$ & $\tau_{n}$ & $\sigma \tau_{n}$ \\
\hline unit & \multicolumn{2}{|c|}{ photon/channel } & \multicolumn{2}{|c|}{ ns } \\
\hline 1 & 15474 & 280 & 3.003 & 0.041 \\
2 & 87361 & 301 & 7.132 & 0.009 \\
\hline
\end{tabular}

Table 6S. Best-fit parameters for unmodified Trp GB1 emission at 385nm, triple-exponential model. $\sigma \alpha_{n}$ and $\sigma \tau_{n}$ represent standard deviations for $\alpha_{n}$ and $\tau_{n}$.

\begin{tabular}{|c|r|r|c|c|}
\hline$n$ & $\alpha_{n}$ & $\sigma \alpha_{n}$ & $\tau_{n}$ & $\sigma \tau_{n}$ \\
\hline unit & \multicolumn{2}{|c|}{ photon/channel } & \multicolumn{2}{|c|}{ ns } \\
\hline 1 & -11694 & 261 & 0.144 & 0.004 \\
2 & 12731 & 942 & 4.109 & 0.122 \\
3 & 51549 & 974 & 7.306 & 0.034 \\
\hline
\end{tabular}


Figure 34S. Reduced-photon-count fluorescence of 5FTrp and unmodified Trp in GB1, measured at two emission wavelengths: $350 \mathrm{~nm}$ (the wavelength used in ref. 24) and $370 \mathrm{~nm}$ (close to the $368 \mathrm{~nm}$ wavelengths used in ref. 25). The data were fitted by the monoexponential model function. The residuals (top) and the data (bottom) are presented in the format of Fig.1 in reference 24 . Not only the format, but also the scales of Fig. 1 in reference 24 were exactly duplicated to facilitate the comparison. Note, that we have reduced the peak photon counts from about $80 \cdot 10^{3}$ (the peak photon count in our typical data) to $7 \cdot 10^{3}$ (the peak counts in ref. 24) for a fair comparison. This corresponds to a reduction in the acquisition time from 24 minutes to about 2 minutes. This figure makes two points: (i) that in this representation it is virtually impossible to detect the minute difference between a monoexponential and a multiexponential kinetic curve, and (ii) that the fluorescence kinetic curves for 5FTrp in GB1 are not fundamentally different than those shown in Fig. 1 in reference 24.

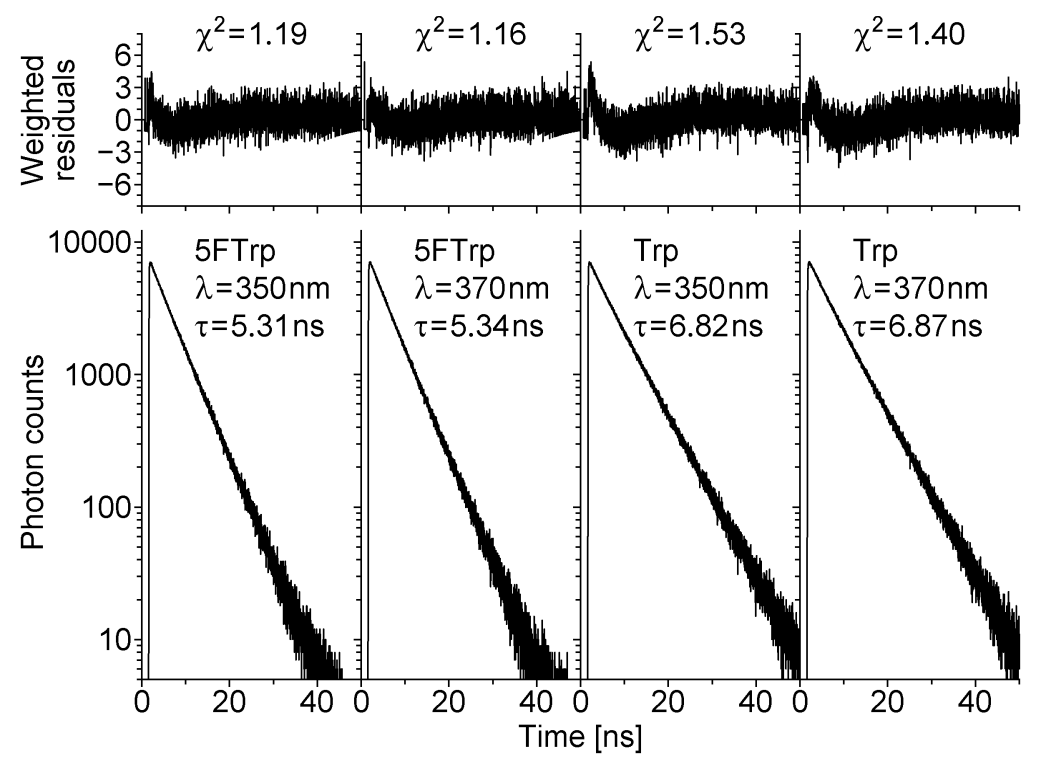


Figure 35S. The dynamics of the time-dependent red shift for Trp and 5FTrp in GB1, without the $t \rightarrow \infty$ limit subtracted. The same data with the $t \rightarrow \infty$ limit subtracted are shown in Fig 4B.

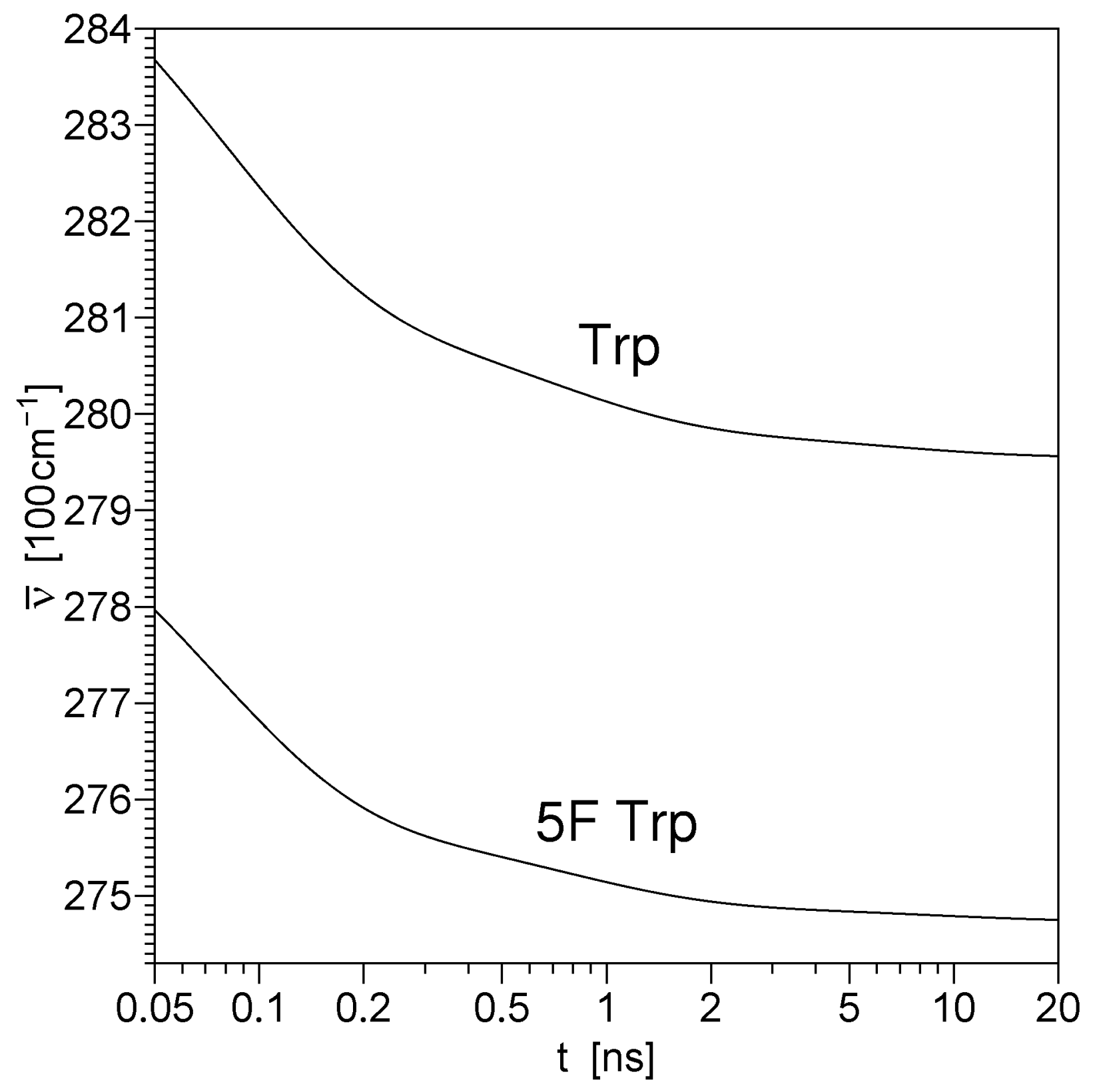


Parametrization of the functions $S(v)$ and $N(t)$ involved in the model function in Eq. (8)

(Global fitting with a model based on parallel translation of the FCF envelope)

Time-Resolved Fitting Function:

$$
F_{n}(t)=\gamma_{n} \Theta(t) P(t) S\left(\lambda_{n}^{-1}-D(t)\right)
$$

$\mathrm{F}_{\mathrm{n}}(\mathrm{t})$ is numerically convoluted with the experimentally-determined IRF and fitted to the timeresolved fluorescence measured at the emission wavelength $\lambda_{n}$.

Indices and parameters involved in time-resolved fitting function only:

$\mathrm{n} \quad \mathrm{n}=1, \ldots, \mathrm{NW}$

NW the Number of the emission Wavelengths at which the time-correlated photons were counted.

$\lambda_{n}$ the emission wavelengths, an array of constants.

$\gamma_{\mathrm{n}} \quad$ scale factors, free fitting parameters.

$\Theta(\mathrm{t})$ Heaviside function.

Steady-State Fitting Function:

$$
F^{S S}(\lambda)=\Gamma \lambda^{-5} \int_{0}^{\infty} P(t) S\left(\lambda^{-1}-D(t)\right) d t
$$

Parameter involved in steady-state fitting function only:

$\Gamma \quad$ scale factor, a free fitting parameter.

Parametrization of the Population Function and the Dynamic Shift Function:

Population:

$$
P(t)=\sum_{i=1}^{N E} \alpha_{i} \exp \left(-t / \tau_{i}\right)
$$

Dynamic Shift:

$$
D(t)=\xi \exp \left[-(\rho t)^{\beta}\right]
$$

Indices and parameters involved in the population and dynamic shift functions:

i $\quad \mathrm{i}=1, \ldots, \mathrm{NE}$

NE the Number of Exponentials.

$\alpha_{i} \quad$ population amplitudes, free fitting parameters.

$\tau_{i} \quad$ population taus, free fitting parameters.

$\xi \quad$ dynamic shift amplitude, a free fitting parameter.

$\rho \quad$ stretched exponential rate constant, a free fitting parameter.

$\beta \quad$ stretched exponential beta constant, free fitting parameters.

Parametrization of the Spectral Shape Function:

$$
S(v)=\exp \left(-\sum_{m=2}^{M P} c_{m}(v-b)^{m}\right)
$$


Indices and parameters involved in the spectral shape function:

$\mathrm{m} \quad \mathrm{m}=2, \ldots, \mathrm{MP}$

MP the Maximum Power of the polynomial.

$\mathrm{Cm}_{\mathrm{m}} \quad$ the coefficient for the power $\mathrm{m}$, a free fitting parameter.

b the peak of $S(v)$, a free fitting parameter.

Numerical calculation of the derivatives of $S(v)$ with respect to $\underline{v}$.

For $\mathrm{k}=0$ :

$$
\frac{\partial^{k}}{\partial v^{k}} S(v)=S(v)
$$

For $k>0$ :

$$
\frac{\partial^{k}}{\partial v^{k}} S(v)=S(v) \sum_{n=0}^{k(M P-1)} d_{k n}(v-b)^{n}
$$

$\underline{\text { Recursive expressions for } \mathrm{d}_{\mathrm{kn}} \text { : }}$

$$
\begin{gathered}
d_{k, n}=(n+1) d_{k-1, n+1}-\sum_{m=2}^{M P} m c_{m} d_{k-1, n+1-m} \\
d_{0, n}=\left[\begin{array}{lll}
1 & \text { if } & n=0 \\
0 & \text { if } & n \neq 0
\end{array}\right]
\end{gathered}
$$

Numerical calculation of the derivatives of $S(v)$ with respect to $v$ and a fitting parameter:

$$
\begin{gathered}
\frac{\partial}{\partial b} \frac{\partial^{k}}{\partial v^{k}} S(v)=-\frac{\partial^{k+1}}{\partial v^{k+1}} S(v) \\
\frac{\partial}{\partial c_{m}} \frac{\partial^{k}}{\partial v^{k}} S(v)=-\sum_{n} \frac{k ! m !}{(k-n) !(m-n) ! n !}(v-b)^{m-n} \frac{\partial^{k-n}}{\partial v^{k-n}} S(v)
\end{gathered}
$$

All numerical calculations in Eqs.(5-9) are carried out by a FORTRAN subroutine DEREXPOL(ARG,CP,MP,DEP,ND,PDDEPCP,IDIM), disk file "derexpol.for" .

Data fitting for JP064528N was done with the following values:

$\mathrm{NW}=31$-- number of wavelengths

$\mathrm{NE}=4 \quad--$ number of exponentials involved in $\mathrm{P}(\mathrm{t})$

$\mathrm{MP}=16$-- maximum power of the polynomial involved in $\mathrm{S}(\mathrm{v})$.

Note: here the definitions of the functions $P(t), S(v)$, and $D(t)$ are slightly different than the definitions of the functions $\mathrm{N}(\mathrm{t}), \mathrm{S}(\mathrm{v})$, and $\bar{v}(t)$ in JP064528N. The definitions used here are more convenient for numerical calculations, but less general than those in JP064528N. 
Figure 36S. Color map of global weighted residuals for the stretched exponential relaxation model.

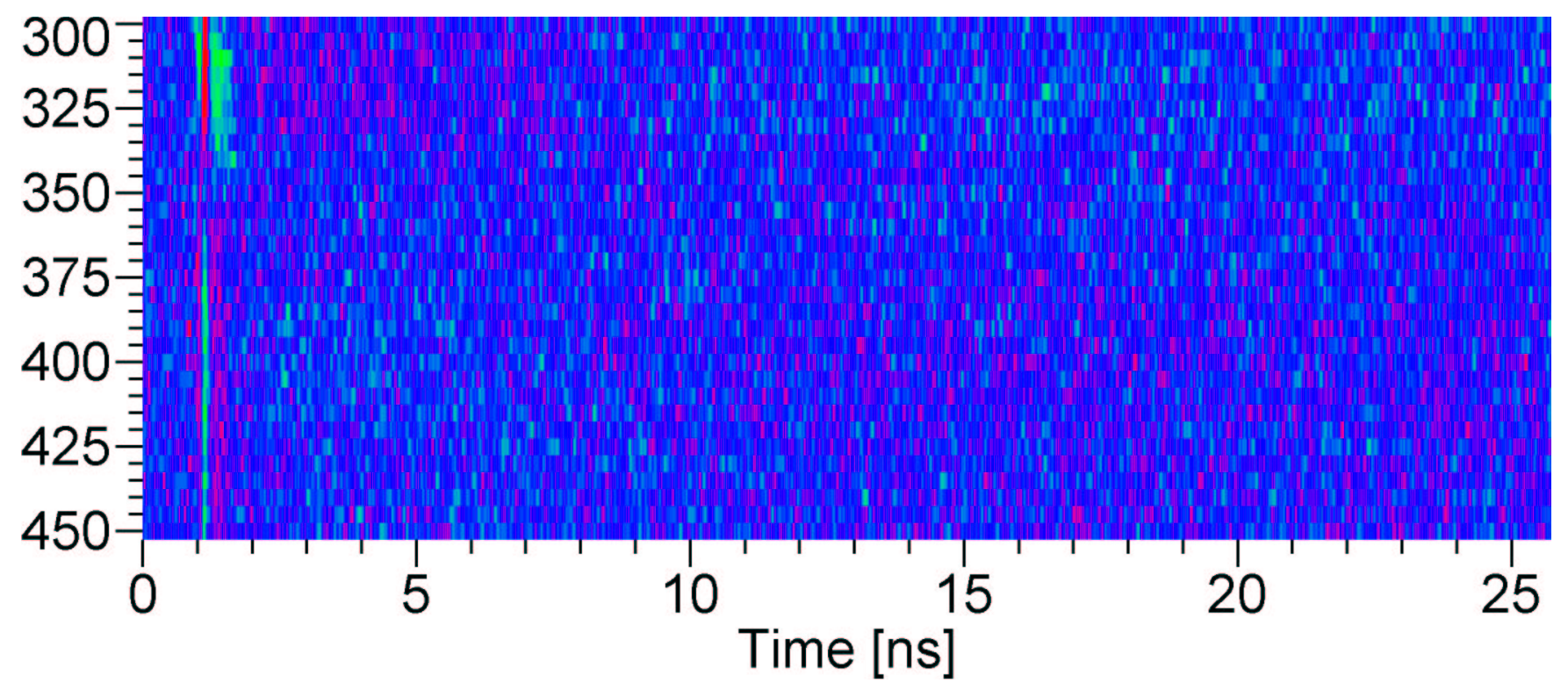

Figure 37S. Color map of global weighted residuals for the triple-exponential relaxation model.

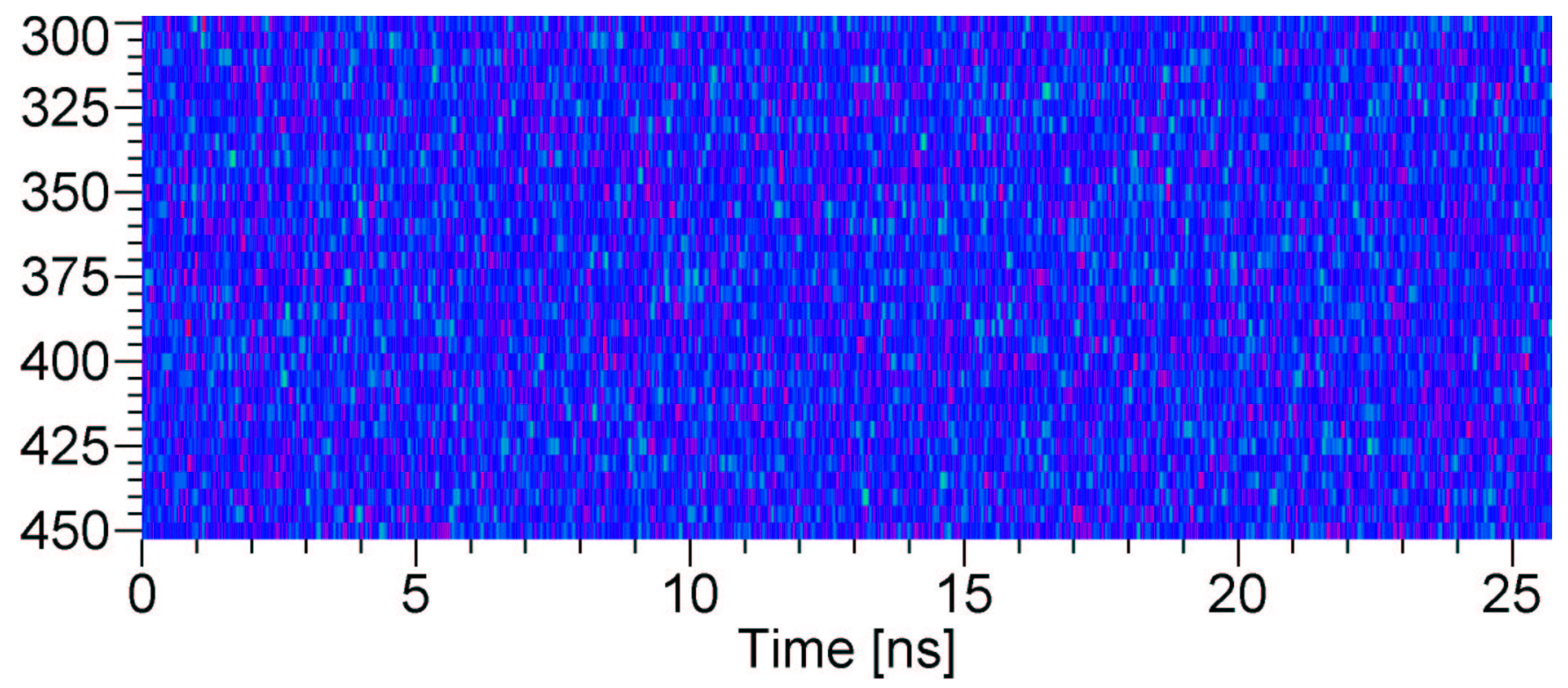

Color scheme:

RED data are significantly greater than the model

MAGENTA data are slightly greater than the model

BLUE data and model are almost equal

CYAN model is slightly greater than the data

GREEN model is significantly greater than the data. 